\title{
Computational method for estimating the domain of attraction of discrete-time uncertain rational systems
}

\author{
Péter Polcz ${ }^{\mathrm{a}, *}$, Tamás Péni ${ }^{\mathrm{b}}$, Gábor Szederkényi ${ }^{\mathrm{a}, \mathrm{b}}$ \\ ${ }^{a}$ Faculty of Information Technology and Bionics, Pázmány Péter Catholic University, \\ Práter u. 50/a, H-1083 Budapest, Hungary \\ ${ }^{b}$ Systems and Control Laboratory, Institute for Computer Science and Control (MTA \\ SZTAKI), Hungarian Academy of Sciences, Kende u. 13-17, H-1111 Budapest, Hungary
}

\begin{abstract}
Using linear matrix inequality (LMI) conditions, we propose a computational method to generate Lyapunov functions and to estimate the domain of attraction (DOA) of uncertain nonlinear (rational) discrete-time systems. The presented method is a discrete-time extension of the approach first presented in (Trofino and Dezuo, 2013), where the authors used Finsler's lemma and affine annihilators to give sufficient LMI conditions for stability. The system representation required for DOA computation is generated systematically by using the linear fractional transformation (LFT). Then a model simplification step not affecting the computed Lyapunov function (LF) is executed on the obtained linear fractional representation (LFR). The LF is computed in a general quadratic form of a state and parameter dependent vector of rational functions, which are generated from the obtained LFR model. The proposed method is compared to the numeric n-dimensional order reduction technique proposed in (D'Andrea and Khatri, 1997). Finally, additional tuning knobs are proposed to obtain more degrees of freedom in the LMI conditions. The method is illustrated on two benchmark examples.
\end{abstract}

Keywords: nonlinear system, Lyapunov function, domain of attraction, linear matrix inequality, model simplification,

\footnotetext{
* Corresponding author

Email addresses: polcz.peter@itk.ppke.hu (Péter Polcz), peni.tamas@sztaki.mta.hu (Tamás Péni), szederkenyi@itk.ppke.hu (Gábor Szederkényi)
} 


\section{Introduction}

Finding or at least approximating the domain of attraction (DOA) of a locally stable equilibrium point of a nonlinear dynamical system is an important but also a non-trivial task in model analysis and controller design/evaluation.

5 This task is most often solved by using a local Lyapunov function (LF), which can determine an invariant stability domain by considering an appropriate level set of the LF. Due to this fact, numerous works have been devoted to the computational construction of LFs, see e.g. [1, 2, 3, 4, 5, where the authors used analytical techniques to iteratively obtain a LF for continuous-time (CT) and/or hybrid switched systems. In [6], an analytical Lyapunov-like solution is proposed for discrete-time (DT) nonlinear dynamical systems by introducing the so-called G-functions. Unlike Lyapunov functions, G-functions do not need to be positive or negative definite. For convergence analysis of DT dynamical systems, [7, 8] used Banach fixed-point principle together with a contraction 15 mapping theorem.

At the same time, there exist alternative numerical Lyapunov-based approaches to determine forward invariant subsets of the state-space, for example, 9, 10] proposed a simulation-guided LF computation method for nonlinear switched and DT systems, respectively, by applying some linear constraints obtained from the execution traces of the dynamics in discrete sample points of a bounded subset of the state-space. Based on multi-resolution state-space sampling approach, [1] considered an initial quadratic finite-step Lyapunov function to systematically find a LF in a general quadratic form of nonlinear terms alongside with a (possibly non-convex) bounded invariant region. The proposed method is applicable for a wide class of DT nonlinear dynamical systems.

Another popular approach for DOA estimation is the so-called sum of squares (SOS) programing. In [12, a rational Lyapunov function and a polynomial static output control law is searched to estimate and manipulate the robust 
DOA for uncertain polynomial systems by solving quasi-convex bilinear matrix inequalities (BMIs), which are formulated from SOS constraints.

It is worth mentioning that the theory of linear matrix inequalities (LMIs) and semidefinite programming (SDP) have made a considerable progress in the last two decades, and together with the useful system modeling technique, the linear fractional transformation (LFT), they provide a powerful framework for 35 stability analysis, robust control and filtering problems. In this field, several new results are available [13, 14, 15. In [16, the authors used a quadratic LF and LFT to represent a rational nonlinear system, and defined convex conditions for stability analysis and state feedback design.

In [17] an LMI approach is presented together with Finsler's lemma, in order 40 to construct polynomial Lyapunov functions for discrete-time nonlinear systems with parameter uncertainties. A recent important result in this line of research is presented in [18, where the authors used Finsler's lemma and the notion of affine annihilators to generate sufficient LMI conditions ensuring local stability for uncertain rational CT systems. The Lyapunov conditions are required only 45 within a bounded polytopic subset of the state-space, therefore, it is enough to check the feasibility of the obtained LMIs only in the corner points of the polytope. Based on this work, [19] analysed the synthesis of sufficient conditions for finite-time stability of nonlinear quadratic systems using polynomial LFs, furthermore, [20] used truncated Taylor expansion to estimate the robust DOA 50 for non-polynomial nonlinear systems.

The results of [18, were developed further in 21, 22], which proposed an LFT-based systematic procedure to construct the required differential-algebraic system representation needed for stability analysis. The authors proposed an efficient method to generate so-called maximal annihilators needed for LMI com55 putations and introduced a model simplification technique, which resulted in a dimensionally reduced optimization problem compared to other known LMIbased solutions in the literature $23,24,25,26,18$.

In this paper, we extend the ideas presented in [18, 21, 22] to discretetime uncertain rational systems. The parameter dependent LF is searched in 
a general quadratic form of rational terms obtained from the linear fractional representation of the dynamic equation. An estimate for the robust DOA is computed within a predefined bounded polytopic subset of the state-space and it is computed as the intersection of the largest level set of the Lyapunov function (inside the polytope) for the different values of the uncertain parameters. To give ${ }_{65}$ sufficient LMI conditions for the required properties of the LF we used Finsler's lemma with maximal annihilators proposed by [21]. The LMI condition for the decreasing property of the LF along the system trajectories is composed in two different ways. Both methods are based on the expansion of the parameter space of the LMI problem by introducing new rational terms into the model.

The paper is organized as follows: Section 2 presents the LMI approach for the computational robust DOA estimation for uncertain nonlinear DT systems. In the next section, we propose an LFR simplification method based on both symbolic and numerical operations. In Section 4, we present two tuning techniques to introduce new degrees of freedom into the second LMI, which implies the decrease of the LF along the system trajectory. In the last section, three benchmark examples are introduced, on which the proposed approach is illustrated and evaluated.

\subsection{Notations, abbreviations}

In this paper, we will use the following notations and abbreviations: $i=\overline{1, n}$ denotes that $i \in\{1, \ldots, n\} .0_{n \times m}$ and $I_{n}$ denote the $n \times m$ zero matrix and the $n \times n$ unit matrix, respectively. We use $A \succ 0$ and $A \prec 0$ to denote that $A \in \mathcal{S}^{m}$ is positive and negative definite, respectively, where $\mathcal{S}^{m}$ denotes the cone of the $m \times m$ symmetric matrices. Given a scalar valued positive definite function $V: \mathbb{R}^{n} \rightarrow \mathbb{R}$, its particular level set $\Omega_{\alpha}=\left\{x \in \mathbb{R}^{n} \mid V(x) \leq \alpha\right\}$ is said to be the $\alpha$-level set of $V(x)$, additionally, $V(x)$ is called proper if $\Omega_{\alpha}$ is a compact set for all $\alpha>0$. Function $f: \mathbb{R}^{n} \rightarrow \mathbb{R}$ is called rational if it can be given as an algebraic fraction of polynomials $p(x)$ and $q(x)$ of the variables $x_{1}, \ldots, x_{n}$, namely $f(x)=f\left(x_{1}, \ldots, x_{n}\right)=\frac{p(x)}{q(x)}$. Furthermore, $f(x)$ is said to be well-defined on $\mathcal{X} \subseteq \mathbb{R}^{n}$ if $q(x) \neq 0$ for all $x \in \mathcal{X}$. We call a polynomial monic if its leading 
coefficient is 1 . The transpose of a matrix $A$ is denoted by $A^{T}$. In this paper, we consider discrete-time (DT) dynamical systems, for which the $k$ th sample of signal $x$ is denoted by $x[k]$. In order to simply represent the dynamics of a system let us use the notation: $x^{+}[k]=x[k+1], k \in \mathbb{N}$.

\subsection{The studied uncertain system class}

In this paper, we consider DT nonlinear systems of the form

$$
x^{+}=f(x, \varrho)=\mathcal{A}(x, \varrho) x,
$$

where $x[k] \in \mathbb{R}^{n}$ is the state vector, $\varrho[k] \in \mathbb{R}^{r}$ is a vector of possibly timedependent uncertain parameters. For simplicity, the time arguments of $x, x^{+}$ and $\varrho$ are suppressed in the sequel. During the stability analysis we assume only a bounded polytopic set of initial conditions $\mathcal{X}$, including the origin $0 \in \mathcal{X}$, furthermore, we assume that the possible values of the uncertain parameter vector $\varrho$ belong to a bounded polytope $\mathcal{P}$. We require that polytopes $\mathcal{X} \subset \mathbb{R}^{n}$, $\mathcal{P} \subset \mathbb{R}^{r}$ be given a priori. Moreover, it is assumed that $f: \mathcal{X} \times \mathcal{P} \rightarrow \mathbb{R}^{n}$ is a vector valued function of $x$ and $\varrho$ having the form

$$
f(x, \varrho)=\left(\begin{array}{c}
f_{1}(x, \varrho) \\
\ldots \\
f_{n}(x, \varrho)
\end{array}\right), \quad f_{i}(x, \varrho)=\sum_{j=1}^{M_{i}} \frac{p_{i j}(x, \varrho)}{q_{i j}(x, \varrho)}, \quad i=\overline{1, n},
$$

${ }_{95}$ where $f_{i}: \mathcal{X} \times \mathcal{P} \rightarrow \mathbb{R}$ are well-defined rational functions on the polytope $\mathcal{X} \times \mathcal{P}, p_{i j}(x, \varrho)$ and $q_{i j}(x, \varrho)$ are polynomials of $(x, \varrho)$ and $q_{i j}(x, \varrho) \neq 0$ for all $(x, \varrho) \in \mathcal{X} \times \mathcal{P}$. We assume that $f(0, \varrho)=0$ for all $\varrho \in \mathcal{P}$, i.e. the origin $x^{*}=0$ is a fixed-point of function $f(\cdot, \varrho)$ for all admissible values of the uncertain parameter $\varrho \in \mathcal{P}$. Consequently, $f(x, \varrho)$ can be written in the form $\mathcal{A}(x, \varrho) x$, where $\mathcal{A}(x, \varrho)$ is a square matrix of rational functions. We assume that the rate of change of the uncertain parameter is bounded, namely, for all $k \in \mathbb{N}$ there exists $\sigma[k] \in \mathcal{R}$ such that $\varrho[k+1]=\varrho[k]+\sigma[k]$, where $\mathcal{R} \in \mathbb{R}^{r}$ is a bounded polytope. With an abuse of notation, we can also write that $\varrho^{+}=\varrho+\sigma$.

We require that the fixed-point $x^{*}=0$ is locally asymptotically stable. The set of all initial conditions, from which the solutions $x[k]$ converge to $x^{*}$ along 
all possible $\varrho[k] \in \mathcal{P}$ and $\sigma[k] \in \mathcal{R}$ trajectories is called the domain of attraction (DOA) $[23$.

\subsection{Model representation}

In order to systematically build up a system representation required for the robust DOA estimation [18, Eq. (16)], the authors of [21] proposed to start with the linear fractional representation (LFR) of the system equation (1). The LFT/LFR is discussed in detail in Chapter 10 of [15] or in the users' manual 27. The nonlinear dynamics of the system equation (1) can be given as follows:

$$
\begin{array}{rlrl}
x^{+} & =A x+B \pi, & & A \in \mathbb{R}^{n \times n}, \quad B \in \mathbb{R}^{n \times p}, \\
y=C x+D \pi, & & C \in \mathbb{R}^{p \times n}, \quad D \in \mathbb{R}^{p \times p}, \\
\pi & =\Delta(x, \varrho) y, & & \Delta(x, \varrho) \in \mathbb{R}^{p \times p},
\end{array}
$$

where Eqs. (3a) and (3b) define a linear time-invariant system with a nonlinear feedback characterized by the uncertain nonlinear square operator $\Delta(x, \varrho)$ in (3c). Matrices $A, B, C$ and $D$ of $(3 \mathrm{a}-\mathrm{b})$ are constant matrices. $x \in \mathbb{R}^{n}$ is the state vector, $\pi, y \in \mathbb{R}^{p}$ represent the feedback signals through the nonlinear uncertain operator $\Delta(x, \varrho)$. In short, we refer to representation $3 \mathrm{a}$-c) as $\mathcal{F}_{l}(A, B, C, D, \Delta)$. In order to give a set of rational functions to consider in the parameterized Lyapunov function, 21] proposed to express variable $\pi \in \mathbb{R}^{p}$ from (3b-c) by eliminating the auxiliary variable $y \in \mathbb{R}^{p}$ :

$$
\begin{aligned}
& G(x, \varrho) x+F(x, \varrho) \pi(x, \varrho)=0, \\
& \text { where } \quad G(x, \varrho)=-\Delta(x, \varrho) C \in \mathbb{R}^{p \times n}, \\
& \\
& F(x, \varrho)=I_{p}-\Delta(x, \varrho) D \in \mathbb{R}^{p \times p} .
\end{aligned}
$$

Hence, we obtain an explicit expression for vector $\pi=\pi(x, \varrho)$, namely

$$
\pi(x, \varrho)=-F^{-1}(x, \varrho) G(x, \varrho) x, \forall(x, \varrho) \in \mathcal{X} \times \mathcal{P} .
$$


We prescribe further algebraic equality constraints:

$$
\begin{aligned}
& N_{b}(x, \varrho) \pi_{b}(x, \varrho)=0, \forall(x, \varrho) \in \mathbb{R}^{n+r}, \\
& \text { where } \pi_{b}(x, \varrho)=\left(\begin{array}{c}
x \\
\pi(x, \varrho)
\end{array}\right) \in \mathbb{R}^{m=n+p},
\end{aligned}
$$

in order to well represent the algebraic interdependence between the state variables $x_{1}, \ldots, x_{n}$ and the nonlinear state and parameter dependent coordinates of vector $\pi(x, \varrho)$. Matrix $N_{b}(x, \varrho) \in \mathbb{R}^{q \times m}$, is a matrix of affine expressions in $x$ and $\varrho$ and is called an affine annihilator. The six equations in (3) give together a model representation, which allows LMI-based LF computation and robust DOA estimation as proposed by [18].

Assumptions. Representation (3) is supposed to fulfill the following assumptions:

(A1) Operator $\Delta(x, \varrho)$ is a diagonal matrix and is affine in the state variables $x_{1}, \ldots, x_{n}$ and in the uncertain parameters $\varrho_{1}, \ldots, \varrho_{r}$. With this regularization, the state and parameter variable matrices $G(x, \varrho)$ and $F(x, \varrho)$ are affine functions of $x$ and $\varrho$, hence, we call them affine matrices.

(A2) Matrix $F(x, \varrho)$ is invertible for all $(x, \varrho) \in \mathcal{X} \times \mathcal{P}$, i.e. the LFR (3a-c) is well-posed.

According to [27], assumption (A2) is an equivalent formulation of that

$$
\mathcal{A}(x, \varrho)=A+B(I-\Delta(x, \varrho) D)^{-1} \Delta(x, \varrho) C
$$

is bounded on $\mathcal{X} \times \mathcal{R}$, namely, the system equation $f(x, \varrho)=\mathcal{A}(x, \varrho) x$ is welldefined on $\mathcal{X} \times \mathcal{R}$.

\subsection{Lyapunov function candidate}

In [18, a suitable LF for representation (3) is searched in the form

$$
V(x, \varrho)=\pi_{b}^{T}(x, \varrho) P \pi_{b}(x, \varrho),
$$

where $P \in \mathbb{R}^{m \times m}$ is a (not necessarily positive definite) symmetric matrix of free parameters. The combined vector $\pi_{b}(x, \varrho)$ defined in (3f) contains the state 
variables and the uncertain rational functions of $\pi(x, \varrho)$, which together determine the structure of the candidate rational parameter dependent Lyapunov function. The difference of the LF along the system trajectories can be given by the following equation

$$
\delta V(x, \varrho, \sigma)=V(f(x, \varrho), \varrho+\sigma)-V(x, \varrho),
$$

where $(x, \varrho, \sigma) \in \mathcal{X} \times \mathcal{P} \times \mathcal{R}$.

The necessary Lyapunov conditions for local stability are the following

$$
\begin{array}{ll}
v_{l}(\|x\|) \leq V(x, \varrho) \leq v_{u}(\|x\|) & \forall(x, \varrho) \in \mathcal{X} \times \mathcal{P}, \\
\delta V(x, \varrho, \sigma) \leq-v_{d}(\|x\|) & \forall(x, \varrho, \sigma) \in \mathcal{X} \times \mathcal{P} \times \mathcal{R},
\end{array}
$$

where $v_{l}, v_{u}$ and $v_{d}$ are strictly increasing continuous scalar functions, being zero in $\|x\|=0$.

Remark 1. Even though the true DOA of the fixed-point $x^{*}$ might be unbounded, the method proposed in this paper assumes that $\mathcal{X}$ and $\mathcal{P}$ are bounded polytopes. Therefore, the computed stability domain, which should be located entirely in the interior of $\mathcal{X}$, will be bounded.

\section{LMI approach to estimate the robust DOA}

The Lyapunov conditions (7) are ensured by sufficient parameter dependent LMI conditions, in which the annihilators (3f) play an important role, namely, they represent equality constraints between the coordinates of $\pi_{b}(x, \varrho)$, therefore, they introduce additional degrees of freedom into the optimization problem through the introduction of some Lagrange multipliers. In this section, we adapt the technique proposed by [18 to discrete-time (DT) systems of the same class described in Section 1.2 and given in representation (3). The main result can be summarized as follows.

Proposition 2.1. Let

$$
V(x, \varrho)=\pi_{b}^{T}(x, \varrho) P \pi_{b}(x, \varrho), P \in \mathbb{R}^{m \times m}, m=n+p
$$


be a rational LF for the system model (3). Then, the change of the LF along the system trajectories can be given in the following form

$$
\delta V(x, \varrho, \sigma)=\pi_{a}^{T}(x, \varrho, \sigma) R \pi_{a}(x, \varrho, \sigma), R \in \mathbb{R}^{m^{\prime} \times m^{\prime}},
$$

where $m^{\prime}=n+2 p, \pi_{a}(x, \varrho, \sigma) \in \mathbb{R}^{m^{\prime}}$ is a vector of rational functions of $x$, $\varrho$ and $\sigma$. Moreover, due to Finsler's lemma [18, 28, [29], the positivity and the negativity of $V(x, \varrho)$ and $\delta V(x, \varrho, \sigma)$, respectively, can be ensured by the following two sufficient affine parameter dependent LMI conditions:

$$
\begin{aligned}
& P+L_{b} N_{b}(x, \varrho)+N_{b}^{T}(x, \varrho) L_{b}^{T} \succ 0, \forall(x, \varrho) \in \mathcal{X} \times \mathcal{P} \\
& R+L_{a} N_{a}(x, \varrho, \sigma)+N_{a}^{T}(x, \varrho, \sigma) L_{a}^{T} \prec 0, \forall(x, \varrho, \sigma) \in \mathcal{X} \times \mathcal{P} \times \mathcal{R}
\end{aligned}
$$

where $P \in \mathbb{R}^{m \times m}, L_{b} \in \mathbb{R}^{m \times q}$ and $L_{a} \in \mathbb{R}^{m^{\prime} \times q^{\prime}}$ are free matrix variables, and the elements of matrix $R=R(P)$ are affine expressions of the free variables in matrix $P$. Finally, $N_{b}(x, \varrho) \in \mathbb{R}^{q \times m}$ and $N_{a}(x, \varrho, \sigma) \in \mathbb{R}^{q^{\prime} \times m^{\prime}}$ are affine annihilators for $\pi_{b}(x, \varrho)$ and $\pi_{a}(x, \varrho, \sigma)$, respectively.

Proof. The fact that (9a) implies $7 \mathrm{a}$ ) is a direct consequence of Finsler's lemma and is also discussed in [18] in Theorem 4.1. In order to derive LMI 9b], we consider the difference equation of the LF with respect to the system dynamics $x^{+}=f(x, \varrho)$, that is

$$
\begin{aligned}
\delta V(x, \varrho, \sigma)= & \pi_{b}^{T}(f(x, \varrho), \varrho+\sigma) P \pi_{b}(f(x, \varrho), \varrho+\sigma) \\
& -\pi_{b}^{T}(x, \varrho) P \pi_{b}(x, \varrho) .
\end{aligned}
$$

Let us introduce the auxiliary vector

$$
\begin{aligned}
& \pi_{a}(x, \varrho, \sigma)=\left(\begin{array}{c}
x \\
\pi(x, \varrho) \\
\pi^{+}(x, \varrho, \sigma)
\end{array}\right) \in \mathbb{R}^{n+2 p}, \\
& \text { with } \pi^{+}(x, \varrho, \sigma)=\pi\left(x^{+}, \varrho^{+}\right)=\pi(f(x, \varrho), \varrho+\sigma) .
\end{aligned}
$$

Then, the right multipliers of $P$ in 10 can be given as follows:

$$
\pi_{b}(f(x, \varrho), \varrho+\sigma)=\left(\begin{array}{c}
A x+B \pi(x, \varrho) \\
\pi^{+}(x, \varrho, \sigma)
\end{array}\right)=A_{a} \pi_{a}(x, \varrho, \sigma)
$$


and

$$
\pi_{b}(x, \varrho)=\left(\begin{array}{c}
x \\
\pi(x, \varrho)
\end{array}\right)=E_{a} \pi_{a}(x, \varrho, \sigma),
$$

where

$$
A_{a}=\left(\begin{array}{ccc}
A & B & 0 \\
0 & 0 & I_{p}
\end{array}\right), E_{a}=\left(\begin{array}{cc}
I_{m} & 0_{p \times m}
\end{array}\right) .
$$

Considering the newly introduced objects in $[13), \delta V(x, \varrho, \sigma)$ can be written in form (8), where

$$
R=A_{a}^{T} P A_{a}-E_{a}^{T} P E_{a}
$$

Now, let $N_{a}(x, \varrho, \sigma)$ be an affine annihilator of $\pi_{a}(x, \varrho, \sigma)$. Then, Finsler's lemma implies that function $\delta V(x, \varrho, \sigma)$ is negative for all $(x, \varrho, \sigma) \in \mathcal{X} \times \mathcal{P} \times \mathcal{R}$ if $(9 \mathrm{~b})$ is satisfied.

Due to the fact that the matrix inequalities in $(9)$ are polytopic LMIs, namely, their expressions are affine in $x, \varrho, \sigma$, which belong to bounded polytopes, it is enough to check the feasibility of (9) only in the corner points of $\mathcal{X} \times \mathcal{P}$ and $\mathcal{X} \times \mathcal{P} \times \mathcal{R}$, respectively.

\subsection{The notion of a maximal affine annihilator}

Due to the equality (3d) of representation (3), a possible annihilator for ${ }_{155} \pi_{b}(x, \varrho)$ can immediately be given by composing the block matrix $C_{b}(x, \varrho)=$ $(G(x, \varrho) \quad F(x, \varrho))$. However, it is shown in [18 that in most cases the application of an additional annihilator $N_{b}(x, \varrho)$ of Eq. (3f) may result in even less conservative LMI conditions, and hence in a better estimate for the DOA. In order to exploit the advantageous properties of Finsler's lemma, the construction of so-called maximal affine annihilators was proposed in 21] This approach can be applied in the discrete-time case as well.

Let $f: \mathbb{R}^{s} \rightarrow \mathbb{R}$ be given in the form $f(w)=\pi^{T}(w) Q \pi(w)$, where $w \in \mathbb{R}^{s}$, $\pi(w) \in \mathbb{R}^{m}$ is a vector of rational functions and $Q \in \mathbb{R}^{m \times m}$ is a symmetric matrix of free parameters. Function $f(w)$ is required to be positive for all 
parameter values $w \neq 0$ belonging to a bounded polytope $\mathcal{W} \in \mathbb{R}^{s}$, which can be ensured by the following (sufficient) affine parameter dependent LMI condition

$$
Q+L N(w)+N^{T}(w) L^{T} \succ 0, \forall w \in \mathcal{W}
$$

where $N(w) \in \mathbb{R}^{q \times m}$ is an affine annihilator of $\pi(w)$, namely, $N(w) \pi(w)=0$, for all $w \in \mathbb{R}^{s}$. We say that $N(w)$ is a maximal affine annihilator of $\pi(w)$ if for any possible affine annihilator $N_{1}(w) \in \mathbb{R}^{q_{1} \times m}$ of $\pi(w)$ the feasible set of the corresponding LMI

$$
Q+L_{1} N_{1}(w)+N_{1}^{T}(w) L_{1}^{T} \succ 0, \forall w \in \mathcal{W}
$$

for matrix $Q$ is contained in the feasible set of the LMI characterized by the maximal annihilator (15). In other words, for any affine annihilator $N_{1}(\omega)$, if a symmetric matrix $Q$ with a certain multiplier $L_{1} \in \mathbb{R}^{m \times q_{1}}$ is a solution for (16), then there exists a matrix $L \in \mathbb{R}^{m \times q}$, such that $Q$ with $L$ is a solution for (15).

From the point of view of the DOA estimation, this means that for a given (fixed) set of rational functions $\pi_{b}(x, \varrho)$ and $\pi_{a}(x, \varrho, \sigma)$ in Eqs. (3f) and (11), using maximal affine annihilators $N_{b}(x, \varrho)$ and $N_{a}(x, \varrho, \sigma)$ in LMIs (9) will lead to the largest DOA estimate, which can be obtained by using affine annihilators. At the same time, an annihilator from a broader class of matrix functions may lead to an even less conservative solution for matrix $P$ of Eq. (5), but the corresponding computation problem is not guaranteed to be solvable in a convex optimization framework.

\subsection{Computing a robust stability domain}

Let us call a given set $X \subset \mathbb{R}^{n}$ a robust stability domain (RSD) of fixed-point $x^{*}$, if the system trajectory converges to $x^{*}$ from any initial condition $x[0] \in X$, for any $\varrho[k] \in \mathcal{P}$ and $\sigma[k] \in \mathcal{R}$ for all $k \in \mathbb{N}$. Note that an RSD is always a subset of the true DOA of a fixed-point $x^{*}$, moreover, the computed RSD can be considered as an estimate of the true DOA. Using a Lyapunov function $V(x, \varrho)$ satisfying (7) a possible RSD can be computed as follows. 
1. First of all, let us define the "hyper" level set of $V(x, \varrho)$ in the extended space $\mathbb{R}^{n+r}$ of $(x, \varrho)$ :

$$
\Omega_{\alpha}=\left\{(x, \varrho) \in \mathbb{R}^{n+r} \mid V(x, \varrho) \leq \alpha\right\}
$$

In Figure 1 , the boundary of this level set $\Omega_{\alpha}$ is illustrated by the orange contour line. Note that every point $(x, \varrho) \in \Omega_{\alpha}$, for which $\varrho \notin \mathcal{P}$, is irrelevant in the stability analysis, therefore, these points may be omitted from of $\Omega_{\alpha}$ and we may introduce the following truncated set (illustrated by the filled orange region in Figure 1]:

$$
\Omega_{\alpha, \mathcal{P}}=\Omega_{\alpha} \cap\left(\mathbb{R}^{n} \times \mathcal{P}\right) .
$$

We can assume that, for a certain value $\alpha$, the truncated level set $\Omega_{\alpha, \mathcal{P}}$ is contained (entirely) in $\mathcal{X} \times \mathcal{P} \subset \mathbb{R}^{n+r}$, therefore, $\Omega_{\alpha, \mathcal{P}}$ is invariant with respect to the system dynamics, namely $(x[0], \varrho[0]) \in \Omega_{\alpha, \mathcal{P}}$ implies that $(x[k], \varrho[k]) \in \Omega_{\alpha, \mathcal{P}}$ for all $k \in \mathbb{N}$. Moreover, according to [18, Corollary 4.1], the feasibility of LMIs (9) imply that for any initial condition $(x[0], \varrho[0]) \in \Omega_{\alpha, \mathcal{P}}$ the system trajectory $x[k]$ will tend exponentially to the fixed-point $x^{*}=0$, for any $\varrho[k] \in \mathcal{P}$ and $\sigma[k] \in \mathcal{R}$, for all $k \in \mathbb{N}$.

2. Secondly, we introduce an auxiliary set, which can be considered as a "projection" of $\Omega_{\alpha, \mathcal{P}}$ onto the subspace of the state variables $\left(\mathbb{R}^{n}\right)$ defined in the following way (Figure 1, blue interval):

$$
\bar{\Omega}_{\alpha, \mathcal{P}}=\left\{x \in \mathbb{R}^{n} \mid \exists \varrho \in \mathcal{P} \text { such that }(x, \varrho) \in \Omega_{\alpha, \mathcal{P}}\right\} .
$$

Note that $\bar{\Omega}_{\alpha, \mathcal{P}} \subset \mathcal{X}$ if $\Omega_{\alpha, \mathcal{P}} \subset \mathcal{X} \times \mathcal{P}$.

3. Finally, we give a robust stability domain for the fixed-point:

$$
\bar{\Omega}_{\alpha, \mathcal{P}}^{x_{0}}=\left\{x \in \mathbb{R}^{n} \mid(x, \varrho) \in \Omega_{\alpha, \mathcal{P}} \text { for all } \varrho \in \mathcal{P}\right\} \subseteq \bar{\Omega}_{\alpha, \mathcal{P}} .
$$

Observe that, for any initial conditions from the $\operatorname{RSD} \bar{\Omega}_{\alpha, \mathcal{P}}^{x_{0}}$, the state vector will remain inside $\bar{\Omega}_{\alpha, \mathcal{P}}$ and will converge to the fixed-point independently of the time evolution of the uncertain parameters. 


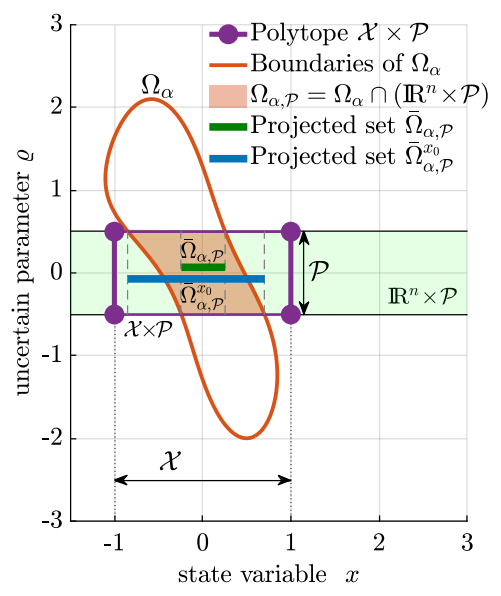

Figure 1: In this figure, we illustrate how the robust stability domain is computed by using a specific level set of the obtained parameter dependent Lyapunov function. For simplicity, the RSD of a first order system is illustrated in this figure with a single uncertain parameter. The orange contour line $\left(\Omega_{\alpha}\right)$ illustrates the $\alpha$ level set of the $\operatorname{LF} V(x, \varrho)$. The light green strip $\left(\mathbb{R}^{n} \times \mathcal{P}\right)$ highlights the region, where $\varrho \in \mathcal{P}$. The filled orange region illustrates the truncated level set $\Omega_{\alpha, \mathcal{P}}$. This truncated set is invariant with respect to the system dynamics. The blue and green intervals illustrate the projected sets $\bar{\Omega}_{\alpha, \mathcal{P}}$ and $\bar{\Omega}_{\alpha, \mathcal{P}}^{x_{0}}$, respectively. If the initial value of the state variable $x[0]$ belongs to the computed robust stability domain $\bar{\Omega}_{\alpha, \mathcal{P}}^{x_{0}}$, the state $x[k]$ will remain inside $\bar{\Omega}_{\alpha, \mathcal{P}}$ for all $k \geq 0$ and will tend to the origin, independently of the uncertain parameter $\varrho[k]$, i.e. $x[k] \in \bar{\Omega}_{\alpha, \mathcal{P}}$ and $x[k] \rightarrow 0$ for all $x[0] \in \bar{\Omega}_{\alpha, \mathcal{P}}^{x_{0}}$ and for all $\varrho[k] \in \mathcal{P}$ such that $\varrho[k+1]-\varrho[k] \in \mathcal{R}$ for all $k \geq 0$. 
In [18, Eqs. (89) and (90)], the authors introduced two further LMI conditions, which ensure that the truncated unitary level set $\Omega_{1, \mathcal{P}}$ is entirely inside of $\mathcal{X} \times \mathcal{P}$. Furthermore, an objective function is proposed to be minimized in order to stretch $\Omega_{1, \mathcal{P}}$ inside $\mathcal{X} \times \mathcal{P}$ as much as possible. From now on, we will consider the truncated unitary level set $\Omega_{1, \mathcal{P}}$ (and the corresponding $\operatorname{RSD} \bar{\Omega}_{1, \mathcal{P}}^{x_{0}}$ ) instead of $\Omega_{\alpha, \mathcal{P}}$.

\section{Symbolic LFR model simplification}

In this section, we present an improved version of the model simplification method for representation (3) proposed by [22], furthermore, we show that the transformed smaller LFR model results in smaller dimensional LMI conditions (9) for stability but giving the same Lyapunov function and computed RSD as the initial higher dimensional LMIs would determine.

\subsection{Non-uniqueness of LFR representation}

It is obvious that the linear fractional representation (3a-c) is not unique for a given system dynamics, and the different representations may result in different Lyapunov functions, thus in different computed robust stability domains. However, in many cases, we can reduce the number of equations in the initial LFR while maintaining the same LF and the same computed RSD. This is obviously advantageous from a computational point of view, since the smaller dimensional LFR results in the same DOA estimate with less computational effort. In order to given an initial LFR for the system equation, we use the symbolic LFT techniques [30, 31, 32, 33] implemented in function sym2lfr of the Enhanced LFR-toolbox for Matlab [34, 27] (LFR-toolbox).

Using our model simplification technique, the initial LFR is considered "reducible" if there exist certain coordinates of the generated combined vector $\pi_{b}(x, \varrho)$, which can be expressed by the linear combination of its other coordinates (with constant coefficients). 
It is worth mentioning that the LFR-toolbox offers to use the numeric n-

like decomposition of matrices $(D, C, B, A)$ of representation $(3 \mathrm{a}-\mathrm{c})$ and eliminates the unobservable and uncontrollable modes from the model. This model transformation results in a so-called relative minimal representation, notion defined in [36, 27. In our RSD computation framework, the model generated by due to the following possible issues:

1. Due to the numeric floating point operations of n-DOR, the obtained LFR $\mathcal{F}_{l}(\breve{A}, \breve{B}, \breve{C}, \breve{D}, \breve{\Delta})$ generates a vector of rational functions $\breve{\pi}(x, \varrho)=$ $(I-\breve{\Delta} \breve{D})^{-1} \breve{\Delta} \breve{C} x$ having a highly complex symbolic representation.

2. As it is demonstrated in [22, Section 5.2], we may lose significant degrees of freedom in the DOA computation if we use n-DOR. Consequently, the reduced model may result in a more conservative estimation for the true DOA.

\subsection{The proposed approach for LFR simplification}

Based on both symbolic and numeric operations, a transformation is proposed in 22] for representation [3], such that certain pairs of variables $\left(\pi_{i}, y_{i}\right)$ can be eliminated from the transformed realization of the initial LFR (3a-c), since they do not affect the system equation (1) of the nonlinear dynamics. Differently from n-DOR, it is not guaranteed that the obtained smaller realization still has advantageous properties for RSD computation. The main advantage of the proposed model simplification method is that it results in a reduced set of functions $\widehat{\pi}(x, \varrho) \in \mathbb{R}^{k}$, which define the same LF (5) as the original vector $\pi(x, \varrho)$. In other words, the smaller LFR results in a smaller dimensional but equivalent LF computation problem.

In this section, we propose an improved model simplification technique based on 22. First of all, a symbolic decomposition of the combined vector $\pi_{b}(x, \varrho)$ 
is considered:

$$
\pi_{b}(x, \varrho)=\frac{1}{q(x, \varrho)} \cdot \Theta \pi_{0}(x, \varrho),
$$

where $\Theta \in \mathbb{R}^{(n+p) \times K}$ is a constant coefficient matrix, $\pi_{0}(x, \varrho)$ is a vector of distinct monic monomials and $q(x, \varrho)$ is the smallest degree common monic denominator of the rational functions in $\pi_{b}(x, \varrho)$. If we disallow $\Theta$ to contain completely zero columns and fix the order of monomials in $\pi_{0}(x, \varrho)$, this decomposition is unique. In order to compute this decomposition, we refer to Section 3 of $[22]$.

Example 1. Consider the following possible value for vector $\pi_{b}(x, \varrho)$. Its decomposition is given by:

$$
\pi_{b}(x, \varrho)=\left(\begin{array}{c}
x_{1} \\
x_{2} \\
\frac{x_{1}}{x_{1}^{2}+4} \\
\frac{x_{1}^{2}}{x_{1}^{2}+4} \\
\frac{x_{1}^{3}}{x_{1}^{2}+4}
\end{array}\right)=\frac{1}{x_{1}^{2}+4} \cdot\left(\begin{array}{ccccc}
1 & 0 & 0 & 4 & 0 \\
0 & 1 & 0 & 0 & 4 \\
\hline 0 & 0 & 0 & 1 & 0 \\
0 & 0 & 1 & 0 & 0 \\
1 & 0 & 0 & 0 & 0
\end{array}\right) \cdot\left(\begin{array}{c}
x_{1}^{3} \\
x_{1}^{2} x_{2} \\
x_{1}^{2} \\
x_{1} \\
x_{2}
\end{array}\right),
$$

dimensions: $n=2, p=3$.

Note that matrix $\Theta$ is rank deficient, and the last element in $\pi_{b}(x, \varrho)$ can be expressed by a linear combination of the other elements, namely

$$
\frac{x_{1}^{3}}{x_{1}^{2}+4}=x_{1}-4 \frac{x_{1}}{x_{1}^{2}+4} .
$$

In this case, we have the possibility to eliminate the redundant element from $\pi_{b}(x, \varrho)$, such that the reduced set of rational functions will define the same algebraic rational structure for the Lyapunov function.

The main results on LFR simplification are summarized in the next proposition, in which we show that if $\Theta$ is rank deficient, we can derive a simplified model representation and solve a smaller dimensional LMI condition to obtain the same Lyapunov function characterizing the same guaranteed robust stability domain of the dynamical system. 
Proposition 3.1. Let us consider a system in representation (3) and a Lyapunov function with the given algebraic structure (5) determined by vector $\pi_{b}(x, \varrho)$ that is considered in the decomposed form (21). We assume that matrix $\Theta$ is rank deficient ( $\operatorname{rank} \Theta=n+k<n+p)$ and the coordinates of vectors $\pi$ and $y$ in representation (3) are written such that the first $n+k$ rows of matrix $\Theta$ are linearly independent.

1. Then, there exist matrices

$$
T_{1} \in \mathbb{R}^{p \times n}, T_{2} \in \mathbb{R}^{p \times k} \text { and } E=\left(\begin{array}{ll}
I_{k} & 0_{k \times(p-k)}
\end{array}\right)
$$

such that the LFR with a smaller dimensional block $\widehat{\Delta}$

$$
\begin{aligned}
x^{+} & =\widehat{A} x+\widehat{B} \widehat{\pi}, \\
\widehat{y} & =\widehat{C} x+\widehat{D} \widehat{\pi}, \\
\widehat{\pi} & =\widehat{\Delta}(x, \varrho) \widehat{y},
\end{aligned}
$$

where

$$
\begin{array}{ll}
\widehat{A}=A+B T_{1}, & \widehat{B}=B T_{2}, \\
\widehat{C}=E C+E D T_{1}, & \widehat{D}=E D T_{2}, \\
\widehat{\Delta}(x, \varrho)=E \Delta(x, \varrho) E^{T}, &
\end{array}
$$

satisfies Assumptions (A1) and (A2) and represents the same dynamics as the initial LFR $3 a-c)$.

2. Furthermore, if we express vector $\widehat{\pi}=\widehat{\pi}(x, \varrho)$ from Eqs. 25b and 25c as

$$
\begin{gathered}
\widehat{\pi}(x, \varrho)=-\widehat{F}^{-1}(x, \varrho) \widehat{G}(x, \varrho) x, \forall(x, \varrho) \in \mathcal{X} \times \mathcal{P}, \\
\text { where } \quad \widehat{G}(x, \varrho)=-\widehat{\Delta}(x, \varrho) \widehat{C} \in \mathbb{R}^{p \times n}, \\
\widehat{F}(x, \varrho)=I_{k}-\widehat{\Delta}(x, \varrho) \widehat{D} \in \mathbb{R}^{p \times p},
\end{gathered}
$$

then, for every symmetric matrix $P \in \mathbb{R}^{(n+p) \times(n+p)}$ there exists a smaller 
symmetric matrix $\widehat{P} \in \mathbb{R}^{(n+k) \times(n+k)}$ such that

$$
\begin{aligned}
& V(x, \varrho)=\pi_{b}^{T}(x, \varrho) P \pi_{b}(x, \varrho)=\widehat{\pi}_{b}^{T}(x, \varrho) \widehat{P} \widehat{\pi}_{b}(x, \varrho), \\
& \text { for all }(x, \varrho) \in \mathbb{R}^{n+r} \text {, where } \widehat{\pi}_{b}(x, \varrho)=\left(\begin{array}{c}
x \\
\widehat{\pi}(x, \varrho)
\end{array}\right) .
\end{aligned}
$$

3. If matrix $P$ is a solution of the parameter dependent LMI

$$
P+L N(x, \varrho)+N^{T}(x, \varrho) L^{T} \succ 0, \forall(x, \varrho) \in \mathcal{X} \times \mathcal{P}
$$

for some matrix $L \in \mathbb{R}^{(n+p) \times q}$, then matrix $\widehat{P}$ of Eq. (26) is a solution of the smaller dimensional LMI

$$
\widehat{P}+\widehat{L} \widehat{N}(x, \varrho)+\widehat{N}^{T}(x, \varrho) \widehat{L}^{T} \succ 0, \forall(x, \varrho) \in \mathcal{X} \times \mathcal{P}
$$

with matrix $\widehat{L}=S^{T} L$ and $\widehat{N}(x, \varrho)=N(x, \varrho) S$, that is an affine annihilator for $\widehat{\pi}_{b}(x, \varrho)$, and

$$
S=\left(\begin{array}{cc}
I_{n} & 0_{n \times k} \\
T_{1} & T_{2}
\end{array}\right) \in \mathbb{R}^{(n+p) \times(n+k)}
$$

is a full column-rank matrix, where $T_{1}$ and $T_{2}$ were introduced in Eq. (24).

4. If the obtained LFR returned by our proposed model simplification method can be further reduced by $n$-DOR, the final relative-minimal model (computed by $n$-DOR) will result in a more conservative LF computation problem.

Proof. 1. Considering decomposition 21], note that matrix $\Theta$ can be written in the following block-matrix form:

$$
\Theta=\left(\begin{array}{c}
\Theta_{x} \\
\Theta_{\pi_{1}} \\
\hline \Theta_{\pi_{2}}
\end{array}\right) \in \mathbb{R}^{(n+p) \times K},
$$

where $\Theta_{x} \in \mathbb{R}^{n \times K}, \Theta_{\pi_{1}} \in \mathbb{R}^{k \times K}, \Theta_{\pi_{2}} \in \mathbb{R}^{(p-k) \times K}$. Correspondingly, we 
can consider a partitioning of system (3a-c):

$$
\begin{aligned}
& x^{+}=A x+B_{1} \pi_{1}+B_{2} \pi_{2}, \\
& y_{1}=C_{1} x+D_{11} \pi_{1}+D_{12} \pi_{2} \in \mathbb{R}^{k}, \\
& y_{2}=C_{2} x+D_{21} \pi_{1}+D_{22} \pi_{2} \in \mathbb{R}^{p-k}, \\
& \pi_{1}=\Delta_{1}(x, \varrho) y_{1} \in \mathbb{R}^{k}, \\
& \pi_{2}=\Delta_{2}(x, \varrho) y_{2} \in \mathbb{R}^{p-k} .
\end{aligned}
$$

Due to the fact that the first $n+k$ rows of $\Theta$ are linearly independent, there exist matrices $\Gamma_{1} \in \mathbb{R}^{(p-k) \times n}$ and $\Gamma_{2} \in \mathbb{R}^{(p-k) \times k}$ such that

$$
\Theta_{\pi_{2}}=\Gamma_{1} \Theta_{x}+\Gamma_{2} \Theta_{\pi_{1}}
$$

Namely, the rows of $\Theta_{\pi_{2}}$ can be expressed as the linear combinations of the rows in matrices $\Theta_{x}$ and $\Theta_{\pi_{1}}$. This directly implies that the explicit expressions of $\pi_{1}$ and $\pi_{2}$ satisfy the following identity:

$$
\pi_{2}(x, \varrho)=\Gamma_{1} x+\Gamma_{2} \pi_{1}(x, \varrho), \text { and } \pi_{b}(x, \varrho)=\left(\begin{array}{c}
x \\
\pi_{1}(x, \varrho) \\
\pi_{2}(x, \varrho)
\end{array}\right) .
$$

The transformation matrices to obtain 25a-c) and 28 can be written as

$$
T_{1}=\left(\begin{array}{c}
0_{k \times n} \\
\Gamma_{1}
\end{array}\right), T_{2}=\left(\begin{array}{c}
I_{k} \\
\Gamma_{2}
\end{array}\right) \stackrel{(29)}{\longrightarrow} S=\left(\begin{array}{c|c}
I_{n} & 0 \\
\hline 0 & I_{k} \\
\Gamma_{1} & \Gamma_{2}
\end{array}\right) .
$$

Using matrix $S$, the original vector $\pi_{b}(x, \varrho)$ can be expressed by the terms of $x$ and $\pi_{1}(x, \varrho)$ as follows:

$$
\pi_{b}(x, \varrho)=S \widehat{\pi}_{b}(x, \varrho), \text { where } \widehat{\pi}_{b}(x, \varrho)=\left(\begin{array}{c}
x \\
\pi_{1}(x, \varrho)
\end{array}\right) .
$$


Substituting $\pi_{2}=\Gamma_{1} x+\Gamma_{2} \pi_{1}$ into Eqs. 31a and (31b), we obtain a reduced LFR:

$$
\begin{aligned}
& x^{+}=\left(A+B_{2} \Gamma_{1}\right) x+\left(B_{1}+B_{2} \Gamma_{2}\right) \pi_{1}, \\
& y_{1}=\left(C_{1}+D_{12} \Gamma_{1}\right) x+\left(D_{11}+D_{12} \Gamma_{2}\right) \pi_{1}, \\
& \text { with } \pi_{1}=\Delta_{1}(x, \varrho) y_{1} .
\end{aligned}
$$

Equations (31c) and 31e can be detached from (36), since in this transformed representation, the system's dynamic equation (36a) does not depend on $\pi_{2}$ and $y_{2}$. Representation (36) describes the same dynamics as the original (decomposed) model (31) with matrices given in (34).

On the other hand, considering the block-matrix decomposition of matrices $G(x, \varrho)$ and $F(x, \varrho)$ of $(3 \mathrm{e})$, equality $(3 \mathrm{~d})$ can be rewritten as follows:

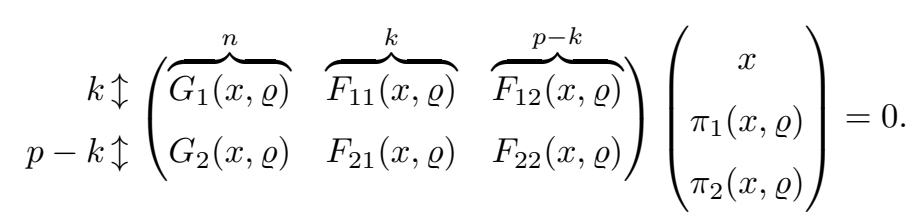

Having (33), and taking only the first $k$ rows of (37), we obtain the following identity:

$$
\widehat{G}(x, \varrho) x+\widehat{F}(x, \varrho) \pi_{1}(x, \varrho)=0,
$$

where

$$
\begin{aligned}
& \widehat{G}(x, \varrho)=G_{1}(x, \varrho)+F_{12}(x, \varrho) \Gamma_{1}, \\
& \widehat{F}(x, \varrho)=F_{11}(x, \varrho)+F_{12}(x, \varrho) \Gamma_{2}=F_{1:}(x, \varrho) T_{2}, \\
& F_{1:}(x, \varrho)=\left(F_{11}(x, \varrho) \quad F_{12}(x, \varrho)\right)
\end{aligned}
$$

Due to the fact that both matrices $F_{1:}(x, \varrho)$ and $T_{2}$ are full row and column rank matrices, respectively, for all $(x, \varrho) \in \mathcal{X} \times \mathcal{R}$, matrix $\widehat{F}(x, \varrho)$ is invertible for all $(x, \varrho) \in \mathcal{X} \times \mathcal{R}$, therefore, vector $\pi_{1}(x, \varrho)$ can be expressed as follows:

$$
\pi_{1}(x, \varrho)=-\widehat{F}^{-1}(x, \varrho) \widehat{G}(x, \varrho) x,
$$

which completes the proof of the first part of Proposition 3.1 . 
2. Let us introduce the block-matrix decomposition of matrix $P$ of the initial Lyapunov function $V(x, \varrho)$ in 26):

$$
P=\left(\begin{array}{ll}
P_{11} & P_{12} \\
P_{21} & P_{22}
\end{array}\right), \text { where } P_{11} \in \mathbb{R}^{(n+k) \times(n+k)} .
$$

Due to 35 , equality 26 can hold if matrix $\widehat{P}$ has the value:

$$
\widehat{P}=S^{T} P S=P_{11}+\Gamma^{T} P_{21}+P_{12} \Gamma+\Gamma^{T} P_{22} \Gamma
$$

where $\Gamma:=\left(\Gamma_{1} \Gamma_{2}\right)$. Thus, the second part of the proposition is proven.

For the sake of completeness, we will show that the two expressions for $\widehat{G}(x, \varrho)$ and $\widehat{F}(x, \varrho)$ in Eqs. (25d) and 39 are equivalent. First of all, we derive the explicit formulas of $G_{i}(x, \varrho), F_{i j}(x, \varrho)$ in the terms of the decomposed matrices of the partitioned representation (31):

$$
\left(\begin{array}{l}
G_{1}(x, \varrho) \\
G_{2}(x, \varrho)
\end{array}\right)=-\left(\begin{array}{cc}
\Delta_{1}(x, \varrho) & 0 \\
0 & \Delta_{2}(x, \varrho)
\end{array}\right)\left(\begin{array}{l}
C_{1} \\
C_{2}
\end{array}\right)=-\left(\begin{array}{l}
\Delta_{1} C_{1} \\
\Delta_{2} C_{2}
\end{array}\right),
$$

and

$$
\left(\begin{array}{cc}
F_{11}(x, \varrho) & F_{12}(x, \varrho) \\
F_{21}(x, \varrho) & F_{22}(x, \varrho)
\end{array}\right)=\left(\begin{array}{cc}
I_{k}-\Delta_{1} D_{11} & -\Delta_{1} D_{12} \\
-\Delta_{2} D_{21} & I_{p-k}-\Delta_{2} D_{22}
\end{array}\right) .
$$

Considering the expressions of matrices $\widehat{C}$ and $\widehat{D}$ of 25$)$, we have that

$$
\begin{aligned}
\widehat{G}(x, \varrho) & =G_{1}(x, \varrho)+F_{12}(x, \varrho) \Gamma_{1} \\
& =-\Delta_{1} C_{1}-\Delta_{1} D_{12} \Gamma_{1}=-\Delta_{1} \widehat{C}, \\
\widehat{F}(x, \varrho) & =F_{11}(x, \varrho)+F_{12}(x, \varrho) \Gamma_{2} \\
& =\left(I_{k}-\Delta_{1} D_{11}\right)-\Delta_{1} D_{12} \Gamma_{2}=I_{k}-\Delta_{1} \widehat{D} .
\end{aligned}
$$

Finally, we obtained the the two definitions 25d and 39 for matrices $\widehat{G}(x, \varrho)$ and $\widehat{F}(x, \varrho)$ are equivalent.

3. Assume that 27 is feasible with $P$ and for some $L$. Since $S$ is full columnrank, the following matrix inequality

$$
S^{T}\left(P+L N(x, \varrho)+N^{T}(x, \varrho) L^{T}\right) S \succ 0
$$

holds for all $(x, \varrho) \in \mathcal{X} \times \mathcal{P}$, which implies 28 . 
4. Let $\mathcal{F}_{l}(\breve{A}, \breve{B}, \breve{C}, \breve{D}, \breve{\Delta})$ denote the relative-minimal LFR computed by the n-DOR and let

$$
\breve{\pi}_{1}=\left(I_{k^{\prime}}-\breve{\Delta} \breve{D}\right)^{-1} \breve{\Delta} \breve{C} x \in \mathbb{R}^{k^{\prime}}, \quad \breve{\pi}_{b}=\left(\begin{array}{c}
x \\
\breve{\pi}_{1}
\end{array}\right) \in \mathbb{R}^{n+k^{\prime}}
$$

denote the corresponding set of rational functions $\left(k^{\prime}<k\right)$.

According to [35, Theorem 4] and [27, Section 2.6], the relative-minimality of $\mathcal{F}_{l}(\breve{A}, \breve{B}, \breve{C}, \breve{D}, \breve{\Delta})$ implies that there exists a similarity transformation for $\mathcal{F}_{l}(\widehat{A}, \widehat{B}, \widehat{C}, \widehat{D}, \widehat{\Delta})$ defined by

$$
\begin{aligned}
& H=\left(\begin{array}{l}
H_{1} \\
H_{2}
\end{array}\right), H^{-1}=\left(\begin{array}{ll}
H_{1}^{\prime} & H_{2}^{\prime}
\end{array}\right) \in \mathbb{R}^{k \times k}, H_{1} \in \mathbb{R}^{k^{\prime} \times k}, \\
& \breve{\pi}=\left(\begin{array}{l}
\breve{\pi}_{1} \\
\breve{\pi}_{2}
\end{array}\right)=H \widehat{\pi}=\left(\begin{array}{l}
H_{1} \widehat{\pi} \\
H_{2} \widehat{\pi}
\end{array}\right),
\end{aligned}
$$

such that the last $k-k^{\prime}$ number of variables $\breve{\pi}$ (namely $\breve{\pi}_{2}$ : the unobservable/uncontrollable modes) of the transformed LFR model

$$
\mathcal{F}_{l}\left(\widehat{A}, \widehat{B} H^{-1}, H \widehat{C}, H \widehat{D} H^{-1}, H \widehat{\Delta} H^{-1}\right)
$$

can be eliminated, since the remaining equations of the transformed LFR (48) represents the same closed-loop system equation $f(x, \varrho)$. If we consider the block matrix decomposition of matrices $H$ and $H^{-1}$ as presented in (47), the relative-minimal representation can be given as:

$$
\begin{aligned}
& \mathcal{F}_{l}(\breve{A}, \breve{B}, \breve{C}, \breve{D}, \breve{\Delta})=\mathcal{F}_{l}\left(\widehat{A}, \widehat{B} H_{1}^{\prime}, H_{1} \widehat{C}, H_{1} \widehat{D} H_{1}^{\prime}, H_{1} \widehat{\Delta} H_{1}^{\prime}\right) \\
& \text { with } \breve{\pi}_{1}=H_{1} \widehat{\pi} .
\end{aligned}
$$

Consequently, vector $\breve{\pi}_{b}(x, \varrho)$ can be expressed as a linear combination of the terms of $\widehat{\pi}_{b}(x, \varrho)$ as follows:

$$
\breve{\pi}_{b}(x, \varrho)=H_{b} \widehat{\pi}_{b}(x, \varrho) \text {, where } H_{b}=\left(\begin{array}{cc}
I_{n} & 0 \\
0 & H_{1}
\end{array}\right) \in \mathbb{R}^{\left(n+k^{\prime}\right) \times(n+k)} .
$$

Since the rational coordinate functions of $\widehat{\pi}_{b}(x, \varrho)$ are (by construction) linearly independent, matrix $H_{b}$ defines a projection of the set of initial 
rational functions onto a smaller set of rational functions. In other words, vector $\widehat{\pi}_{b}(x, \varrho)$ cannot be retained from the projected vector $\breve{\pi}_{b}(x, \varrho)$ (as the initial vector $\pi_{b}(x, \varrho)$ can be expressed by $\widehat{\pi}_{b}(x, \varrho)$ in Eq. $(35)$ ), therefore, the LF

$$
\breve{V}(x, \varrho)=\widehat{\pi}_{b}^{T}(x, \varrho) H_{b}^{T} \breve{P} H_{b} \widehat{\pi}_{b}(x, \varrho),
$$

with the symmetric matrix $\breve{P} \in \mathbb{R}^{k^{\prime} \times k^{\prime}}$ of free parameters contains less linearly independent rational terms than the initial LF. This clearly results in a more conservative (or at least not in a larger) DOA estimation.

\section{Tuning knobs}

In the following motivating example, we demonstrate that the obtained vector $\pi_{a}(x, \varrho, \sigma)$ (defined in Eq. (11)), which contains the rational terms appearing in $\delta V(x, \varrho, \sigma)$, may be improved in the sense that the corresponding annihilator $N_{a}(x, \varrho, \sigma)$ represents more algebraic relations between the coordinates of $\pi_{a}(x, \varrho, \sigma)$. The following simple example illustrates such a case.

Example 2. Consider the following trivial dynamical system

$$
x^{+}=x^{3}, x[0] \in \mathcal{X}=[-a, a],
$$

where $a \in(0,1)$ is a constant. Due to the fact that $|x|^{3}<|x|$ for all $x \in \mathcal{X}$ the solution will converge exponentially to the locally asymptotically stable fixedpoint $x^{*}=0$. Using LFT, the system equation 52 is given in representation (3), with the following model matrices $(A, B, C, D, \Delta)$ and vector $\pi$ :

$$
\begin{aligned}
& \left(\begin{array}{l|l}
A & B \\
C & D
\end{array}\right)=\left(\begin{array}{l|ll}
0 & 0 & 1 \\
\hline 1 & 0 & 0 \\
0 & 1 & 0
\end{array}\right), \Delta(x)=\left(\begin{array}{ll}
x & 0 \\
0 & x
\end{array}\right), \\
& \pi(x)=\left(\begin{array}{c}
x^{2} \\
x^{3}
\end{array}\right), \pi_{b}(x)=\left(\begin{array}{c}
x \\
x^{2} \\
x^{3}
\end{array}\right) .
\end{aligned}
$$


For this model, the LF is searched in form (5). The difference of the LF can be written in form (8), where vector $\pi_{a}$ and its corresponding maximal affine annihilator are the following:

$$
N_{a}(x)=\left(\begin{array}{ccccc}
-x & 1 & 0 & 0 & 0 \\
0 & -x & 1 & 0 & 0
\end{array}\right), \pi_{a}(x)=\left(\begin{array}{c}
x \\
\pi(x) \\
\pi^{+}(x)
\end{array}\right)=\left(\begin{array}{c}
x \\
x^{2} \\
x^{3} \\
\hline x^{6} \\
x^{9}
\end{array}\right) .
$$

Note that in case of using an affine annihilator, the algebraic interdependence between the functions of $\pi^{+}(x)$ and $\pi_{b}(x)$ cannot be expressed, due to the difference between the exponents of the monomials in $\pi_{a}(x)$. In other words, the affine matrix $N_{a}(x)$ is an annihilator for a wide class of functions

$$
z_{a}(x)=\left(\begin{array}{c}
x \\
x^{2} \\
x^{3} \\
z_{4}(x) \\
z_{5}(x)
\end{array}\right),
$$

where $z_{4}(x)$ and $z_{5}(x)$ can be arbitrary scalar functions, since their algebraic expression is not fixed relatively to each other or to the remaining coordinates of $\pi_{a}(x)$.

If we introduce some additional monomials into $\pi_{a}(x)$, such as $x^{4}, x^{5}, x^{7}$, $x^{8}$, we can generate a more representative affine annihilator

$$
\widetilde{N}_{a}(x)=\left(\begin{array}{ccccc|cccc}
x & -1 & 0 & 0 & 0 & 0 & 0 & 0 & 0 \\
0 & x & -1 & 0 & 0 & 0 & 0 & 0 & 0 \\
0 & 0 & x & 0 & 0 & -1 & 0 & 0 & 0 \\
0 & 0 & 0 & 1 & 0 & 0 & -x & 0 & 0 \\
0 & 0 & 0 & x & 0 & 0 & 0 & -1 & 0 \\
0 & 0 & 0 & 0 & 1 & 0 & 0 & 0 & -x \\
0 & 0 & 0 & 0 & 0 & x & -1 & 0 & 0 \\
0 & 0 & 0 & 0 & 0 & 0 & 0 & x & -1
\end{array}\right) .
$$


such that the symbolic matrix multiplication $\widetilde{N}_{a}(x) \widetilde{\pi}_{a}(x)$ gives zero for a more limited set of vectors $\widetilde{z}_{a}(x)$, where $\widetilde{\pi}_{a}(x)$ and $\widetilde{z}_{a}(x)$ denote the augmented vectors

$$
\widetilde{\pi}_{a}(x)=\left(\begin{array}{c}
x \\
x^{2} \\
x^{3} \\
x^{6} \\
x^{9} \\
x^{4} \\
x^{5} \\
x^{7} \\
x^{8}
\end{array}\right), \quad \widetilde{z}_{a}(x)=\left(\begin{array}{c}
x \\
x^{2} \\
x^{3} \\
z_{4}(x) \\
z_{5}(x) \\
x^{4} \\
x^{5} \\
x^{7} \\
x^{8}
\end{array}\right) .
$$

Therefore, $\widetilde{N}_{a}(x)$ can result in a higher dimensional but less conservative sufficient LMI condition $9 \mathrm{~b}$ ) for the negativity of $\delta V(x, \varrho, \sigma)$.

Remark 2. Typically non-advantageous annihilators are those having completely zero columns but also the block diagonal matrices, e.g.

$$
N_{a}(x)=\left(\begin{array}{cc|cc}
-x & 1 & 0 & 0 \\
\hline 0 & 0 & -x & 1
\end{array}\right), \pi_{a}(x)=\left(\begin{array}{c}
x \\
x^{2} \\
x^{5} \\
x^{6}
\end{array}\right), z_{a, k}(x)=\left(\begin{array}{c}
x \\
x^{2} \\
x^{k} \\
x^{k+1}
\end{array}\right) .
$$

One can easily check that $N_{a}(x) \cdot z_{a, k}(x)$ gives zero vector for any $k \in \mathbb{N}$.

Based on the above observations, we can state the problem of improving an annihilator by adding further coordinates to $\pi_{a}(x, \varrho, \sigma)$.

Problem statement. Assume the structure of $\pi_{a}(x, \varrho, \sigma)$ is such that the corresponding annihilator $N_{a}(x, \varrho, \sigma)$ does not represent the algebraic interdependence between the rational/polynomial terms in $\pi_{a}(x, \varrho, \sigma)$, namely, the obtained affine matrix $N_{a}(x, \varrho, \sigma)$ is an appropriate annihilator for a large set of vectors $z_{a}(x, \varrho, \sigma)$ different from $\pi_{a}(x, \varrho, \sigma)$. In this section, we present two different techniques how to supplement the initial vector $\pi_{a}(x, \varrho, \sigma)$, such that the corresponding annihilator results in a less conservative LMI. 


\subsection{Method I. Difference based approach}

310

In [18, Theorem 4.1] the derivative of expression $N(x, \varrho) \pi_{b}(x, \varrho)=0$ is computed in order to generate a new vector $\bar{\pi}_{a}(x, \varrho, \sigma)$, in which further new rational functions would appear. Furthermore, a closed formula is given for a possible annihilator of $\bar{\pi}_{a}(x, \varrho, \sigma)$, which is successfully applied to compute forward invariant domain for continuous-time nonlinear systems. In this section, we adopt this method for discrete-time systems.

We propose to introduce the following new rational functions into $\pi_{a}(x, \varrho, \sigma)$ :

$$
\begin{aligned}
& \bar{\pi}_{a}(x, \varrho, \sigma)=\left(\begin{array}{c}
\pi_{a}(x, \varrho, \sigma) \\
\mu(x, \varrho, \sigma)
\end{array}\right), \\
& \text { where } \mu(x, \varrho, \sigma)=\left(\begin{array}{c}
\mu_{1}(x, \varrho, \sigma) \\
\ldots \\
\mu_{n}(x, \varrho, \sigma)
\end{array}\right) \in \mathbb{R}^{n^{2}+2 n p}, \\
& \text { and } \mu_{k}(x, \varrho, \sigma)=x_{k}^{+} \pi_{a}(x, \varrho, \sigma) \in \mathbb{R}^{n+2 p}, k=\overline{1, n} .
\end{aligned}
$$

Due to this construction, the difference of the LF can be written in the terms of the new set of rational functions, namely:

$$
\begin{gathered}
\delta V(x, \varrho, \sigma)=\bar{\pi}_{a}^{T}(x, \varrho, \sigma)\left(H^{T} R H\right) \bar{\pi}_{a}(x, \varrho, \sigma), \\
\text { where } H=\left(\begin{array}{ll}
I_{n+2 p} & 0_{(n+2 p) \times\left(n^{2}+2 n p\right)}
\end{array}\right) .
\end{gathered}
$$

The construction of the annihilator of the modified vector $\bar{\pi}_{a}(x, \varrho, \sigma)$ is presented in the following proposition.

Proposition 4.1. Let $N(x, \varrho)$ be an affine annihilator for $\pi_{b}(x, \varrho)$ that is decomposed as follows:

$$
N(x, \varrho)=\sum_{k=1}^{n} N_{k} x_{k}+N_{0}(\varrho) \in \mathbb{R}^{q \times m},
$$

where $N_{k}$ are constant matrices and $N_{0}(\varrho)$ is an affine matrix valued function of $\varrho$. Furthermore, let $N_{a}(x, \varrho, \sigma) \in \mathbb{R}^{q^{\prime} \times m^{\prime}}$ be an affine annihilator of the initial vector $\pi_{a}(x, \varrho, \sigma)$. Then, an annihilator for $\bar{\pi}_{a}(x, \varrho, \sigma)$ can be constructed as 
follows:

$$
\begin{aligned}
& \aleph_{\bar{\pi}_{a}}(x, \varrho, \sigma)=\left(\begin{array}{cc}
N_{a}(x, \varrho, \sigma) & 0_{q^{\prime} \times\left(n^{2}+2 n p\right)} \\
0_{n q^{\prime} \times(n+2 p)} & W_{2}(x, \varrho, \sigma) \\
N_{0}\left(\varrho^{+}\right) A_{a} & W_{3} \\
W_{41}(x) & W_{42}
\end{array}\right), \\
& \aleph_{\bar{\pi}_{a}}(x, \varrho, \sigma) \in \mathbb{R}^{\left(q^{\prime}+n q^{\prime}+q+n^{2}\right) \times(n+1)(n+2 p)},
\end{aligned}
$$

where $\varrho^{+}=\varrho+\sigma$ and

$$
\begin{aligned}
& W_{2}(x, \varrho, \sigma)=I_{n} \otimes N_{a}(x, \varrho, \sigma), \\
& W_{3}=\left(\begin{array}{llll}
N_{1} A_{a} & N_{2} A_{a} & \ldots & N_{n} A_{a}
\end{array}\right), \\
& W_{41}(x)=\left(I_{n} \otimes x\right) \cdot\left(\begin{array}{lll}
A & B & 0_{n \times p}
\end{array}\right), \\
& W_{42}=-I_{n} \otimes\left(\begin{array}{ll}
I_{n} & 0_{n \times 2 p}
\end{array}\right) .
\end{aligned}
$$

In 62 , operator $\otimes$ denotes the Kronecker product.

Proof. For the sake of simplicity, the arguments of vectors $\pi, \pi_{b}, \pi_{a}, \mu$ will be suppressed in this proof. Affine matrix $N_{a}(x, \varrho, \sigma)$ is an annihilator of $\pi_{a}$ and hence of $\mu_{k}$ for all $k=\overline{1, n}$. On the other hand, if we compute the difference of $N(x, \varrho) \pi_{b}(x, \varrho)$ and considering that $\pi_{b}^{+}=A_{a} \pi_{a}$, we obtain the following identity:

$$
\begin{aligned}
\delta\left(N(x, \varrho) \pi_{b}\right) & =N(x, \varrho)^{+} \pi_{b}^{+}-N(x, \varrho) \pi_{b} \\
& =N_{0}\left(\varrho^{+}\right) A_{a} \pi_{a}+\sum_{k=1}^{n} N_{k} A_{a} \mu_{k} \\
& =N_{0}\left(\varrho^{+}\right) A_{a} \pi_{a}+W_{3} \mu=0 .
\end{aligned}
$$

Finally, we can observe that

$$
\left(\begin{array}{ll}
I_{n} & 0_{n \times 2 p}
\end{array}\right) \mu_{k}=x x_{k}^{+},
$$

therefore, if we collect vectors $x x_{k}^{+}$into a composed vector, we obtain an affine 
relationship between $\mu$ and $\pi_{a}$ :

$$
\begin{aligned}
{\left[I_{n} \otimes\left(\begin{array}{ll}
I_{n} & 0_{n \times 2 p}
\end{array}\right)\right] \cdot \mu } & =\left(\begin{array}{c}
x x_{1}^{+} \\
\ldots \\
x x_{n}^{+}
\end{array}\right)=\left(I_{n} \otimes x\right) x^{+} \\
& =\left(I_{n} \otimes x\right) \cdot\left(\begin{array}{lll}
A & B & 0_{n \times p}
\end{array}\right) \pi_{a} .
\end{aligned}
$$

Identity 65 gives the last row of annihilator $\boldsymbol{\aleph}_{\bar{\pi}_{a}}(x, \varrho, \sigma)$.

In most cases, the newly introduced functions $\mu_{k}(x, \varrho, \sigma)$ in vector $\bar{\pi}_{a}(x, \varrho, \sigma)$ result in a more representative annihilator $\boldsymbol{\aleph}_{\bar{\pi}_{a}}(x, \varrho, \sigma)$, thus, the LMI $9 \mathrm{~b}$ ) corresponding to the new vector $\bar{\pi}_{a}(x, \varrho, \sigma)$ may result in a better estimate of the DOA. Another advantage of this technique is that the introduction of vector $\mu$ entails a closed formula for a possible annihilator for the augmented vector ${ }_{325} \bar{\pi}_{a}(x, \varrho, \sigma)$, although, annihilator $\boldsymbol{\aleph}_{\bar{\pi}_{a}}(x, \varrho, \sigma)$ may not be maximal. At the same time, the $n^{2}+2 n p$ number of newly introduced functions in $\bar{\pi}_{a}(x, \varrho, \sigma)$ constitute a significant increase in the dimension of the second LMI condition (9b), especially in the case of a large number of nonlinear functions in vector $\pi(x, \varrho)$.

Example 2 (continued). If we apply Method I on the objects $N_{a}(x)$ and $\pi_{a}(x)$ in (52) computed for the demonstrative model $x^{+}=x^{3}$ of Example2 2 we obtain the following vector $\bar{\pi}_{a}^{(1)}(x)$ and its corresponding annihilator $\bar{N}_{a}^{(1)}(x)$ :

$$
\begin{aligned}
& \bar{N}_{a}^{(1)}(x)=\left(\begin{array}{ccccc|ccccc}
x & -1 & 0 & 0 & 0 & 0 & 0 & 0 & 0 & 0 \\
0 & x & -1 & 0 & 0 & 0 & 0 & 0 & 0 & 0 \\
0 & 0 & 0 & 0 & 0 & x & -1 & 0 & 0 & 0 \\
0 & 0 & 0 & 0 & 0 & 0 & x & -1 & 0 & 0 \\
0 & 0 & 0 & -1 & 0 & 0 & 0 & 1 & 0 & 0 \\
0 & 0 & 0 & 0 & -1 & 0 & 0 & 0 & 1 & 0 \\
0 & 0 & x & 0 & 0 & -1 & 0 & 0 & 0 & 0
\end{array}\right), \\
& \bar{\pi}_{a}^{(1)}(x)=\left(\begin{array}{lllll|lllll}
x & x^{2} & x^{3} & x^{6} & x^{9} & x^{4} & x^{5} & x^{6} & x^{9} & x^{12}
\end{array}\right)^{T} .
\end{aligned}
$$

One can observe that three new monomials are introduced: $x^{4}, x^{5}$ and $x^{12}$. The first two monomials make possible to represent the interdependence between $x^{3}$ 
and $x^{6}$. Note that this model can be further simplified by using the proposed method presented in Section 3 , since some functions appear multiple times in the obtained vector $\bar{\pi}_{a}^{(1)}(x)$. In other words, the coefficient matrix $\Theta$ of the obtained vector $\bar{\pi}_{a}^{(1)}(x)$ is rank deficient. Furthermore, according to 21, Section 4.1], a certain number of rows can be eliminated from the modified annihilator $\bar{N}_{a}^{(1)}(x)$ without increasing the conservatism of the RSD computation problem. The reduced model is the following:

$$
\begin{aligned}
& \bar{N}_{a}(x)=\left(\begin{array}{ccccc|ccc}
x & -1 & 0 & 0 & 0 & 0 & 0 & 0 \\
0 & x & -1 & 0 & 0 & 0 & 0 & 0 \\
0 & 0 & 0 & 0 & 0 & x & -1 & 0 \\
0 & 0 & 0 & -1 & 0 & 0 & x & 0 \\
0 & 0 & x & 0 & 0 & -1 & 0 & 0
\end{array}\right)^{T}, \\
& \bar{\pi}_{a}(x)=\left(\begin{array}{lllll|lll}
x & x^{2} & x^{3} & x^{6} & x^{9} & x^{4} & x^{5} & x^{12}
\end{array}\right)^{T} .
\end{aligned}
$$

This annihilator $\bar{N}_{a}(x)$ still has an unfortunate structure, since it has two zero columns, but now, monomial $x^{6}$ is well-represented due to the appearance of (the initially missing) monomials $x^{4}$ and $x^{5}$.

\subsection{Method II. LFT based approach}

In this subsection, we select new rational functions to supplement the initial set of functions in $\pi_{a}(x, \varrho, \sigma)$ by using the linear fractional transformation. This approach guarantees that the finally obtained supplemented vector (denoted by $\left.\widetilde{\pi}_{a}(x, \varrho, \sigma)\right)$ will have a maximal annihilator $\widetilde{N}_{a}(x, \varrho, \sigma)$, which has in each row at least one nonzero element. Let us consider the linear fractional representation of vector $\pi_{a}(x, \varrho, \sigma)$ as follows:

$$
\begin{aligned}
\pi_{a} & =H_{1} x+H_{2} \widetilde{\pi}, \quad \text { let } \widetilde{\pi}_{a}=\left(\begin{array}{c}
x \\
\widetilde{\pi}
\end{array}\right), \\
z & =T_{1} x+T_{2} \widetilde{\pi}, \\
\widetilde{\pi} & =\Delta(x, \varrho, \sigma) z, \quad \text { with } \Delta(x, \varrho, \sigma) \in \mathbb{R}^{p^{\prime} \times p^{\prime}} .
\end{aligned}
$$


Using the second two equations of 68a), one can obtain the following explicit expression for $\tilde{\pi}$ :

$$
\begin{gathered}
\tilde{\pi}(x, \varrho, \sigma)=-F_{a}^{-1}(x, \varrho, \sigma) G_{a}(x, \varrho, \sigma) x, \\
\text { where } F_{a}(x, \varrho, \sigma)=I-\Delta(x, \varrho, \sigma) T_{2}, \\
G_{a}(x, \varrho, \sigma)=-\Delta(x, \varrho, \sigma) T_{1},
\end{gathered}
$$

which also entails the following affine expression between the nonlinear coordinate functions of vector $\widetilde{\pi}_{a}(x, \varrho, \sigma)$ :

$$
\left(G_{a}(x, \varrho, \sigma) \quad F_{a}(x, \varrho, \sigma)\right) \cdot \widetilde{\pi}_{a}(x, \varrho, \sigma)=0 .
$$

In order to eliminate the linearly dependent coordinate functions from vector $\widetilde{\pi}_{a}(x, \varrho, \sigma)$, we applied again the proposed LFR simplification method described in Section 3 to the LFR (68a). Without the loss of generality, we can assume that representation $(68 \mathrm{a})$ is already in its simplified form.

Using the terms of the new vector $\widetilde{\pi}_{a}(x, \varrho, \sigma)$, function $\delta V(x, \varrho, \sigma)$ can be expressed as follows:

$$
\begin{aligned}
& \delta V(x, \varrho, \sigma)=\tilde{\pi}_{a}^{T}(x, \varrho, \sigma)\left(H^{T} R H\right) \tilde{\pi}_{a}(x, \varrho, \sigma), \\
& \text { where } H=\left(\begin{array}{ll}
H_{1} & H_{2}
\end{array}\right) .
\end{aligned}
$$

It is important to mention that, in most practical examples, $\widetilde{\pi}_{a}(x, \varrho, \sigma)$ is typically larger dimensional than $\bar{\pi}_{a}(x, \varrho, \sigma)$, however, due to the attributes of the LFR, we can assure that there exists an annihilator for $\widetilde{\pi}_{a}(x, \varrho, \sigma)$, in which each 340 column contains nonzero elements. This result is summarized in the following proposition.

Proposition 4.2. Assume that the LFR (68a) is well-posed, namely matrix $F_{a}(x, \varrho, \sigma)$ is invertible. Then, vector $\widetilde{\pi}_{a}(x, \varrho, \sigma)$ admits a maximal annihilator $\widetilde{N}_{a}(x, \varrho, \sigma)$, in which every row contains nonzero elements.

Proof. If $n \geq 2$, a possible annihilator for $\widetilde{\pi}_{a}(x, \varrho, \sigma)$ can be given as follows:

$$
\begin{aligned}
& \boldsymbol{\aleph}_{\widetilde{\pi}_{a}}(x, \varrho, \sigma)=\left(\begin{array}{ccc}
\boldsymbol{\aleph}_{x} & 0_{(n-1) \times p^{\prime}} \\
G_{a}(x, \varrho, \sigma) & F_{a}(x, \varrho, \sigma)
\end{array}\right), \\
& \text { where } \boldsymbol{\aleph}_{x}=\left(\begin{array}{cccc}
x_{2} & -x_{1} & \ldots & 0 \\
0 & \cdots & \dddot{x}_{n} & -x_{n-1}
\end{array}\right) \in \mathbb{R}^{n-1 \times n} .
\end{aligned}
$$


345 The invertibility of $F_{a}(x, \varrho, \sigma)$ implies that each column of $\boldsymbol{\aleph}_{\widetilde{\pi}_{a}}(x, \varrho, \sigma)$ contains at least one nonzero element. Additionally, each row of matrix $\boldsymbol{\aleph}_{\widetilde{\pi}_{a}}(x, \varrho, \sigma)$ can be given as the linear combination of the rows of a maximal affine annihilator $\widetilde{N}_{a}(x, \varrho, \sigma)$ of $\widetilde{\pi}_{a}(x, \varrho, \sigma)$. Finally we have that each column of $\widetilde{N}_{a}(x, \varrho, \sigma)$ has nonzero elements. If $n=1$, it is easy to show that matrix $T_{1} \in \mathbb{R}^{p^{\prime} \times 1}$ of Eq. 68a and hence matrix $G_{a}(x, \varrho, \sigma)$ must be full rank, otherwise, according to $68 \mathrm{~b}, \tilde{\pi}_{a}(x, \varrho, \sigma)$ would be zero.

As a consequence of Proposition 4.2, we can say that each coordinate of vector $\tilde{\pi}_{a}(x, \varrho, \sigma)$ is algebraically related to another coordinate of $\tilde{\pi}_{a}(x, \varrho, \sigma)$ given by the corresponding row of $\widetilde{N}_{a}(x, \varrho, \sigma)$.

Example 2 (continued). The generated vector $\tilde{\pi}_{a}(x)$ and its corresponding maximal annihilator are illustrated below for the system $x^{+}=x^{3}$ of Example 2.

$$
\begin{gathered}
\widetilde{N}_{a}(x)=\left(\begin{array}{ccccccccc}
x & -1 & 0 & 0 & 0 & 0 & 0 & 0 & 0 \\
0 & x & -1 & 0 & 0 & 0 & 0 & 0 & 0 \\
0 & 0 & x & 0 & 0 & -1 & 0 & 0 & 0 \\
0 & 0 & 0 & 1 & -x & 0 & 0 & 0 & 0 \\
0 & 0 & 0 & x & 0 & 0 & 0 & 0 & -1 \\
0 & 0 & 0 & 0 & 1 & -x & 0 & 0 & 0 \\
0 & 0 & 0 & 0 & 0 & 0 & 1 & -x & 0 \\
0 & 0 & 0 & 0 & 0 & 0 & 0 & 1 & -x
\end{array}\right), \\
\tilde{\pi}_{a}(x)=\left(\begin{array}{ccccccccc}
x & x^{2} & x^{3} & x^{6} & x^{5} & x^{4} & x^{9} & x^{8} & x^{7}
\end{array}\right)^{T} .
\end{gathered}
$$

Observe that the LFT generated a vector $\widetilde{\pi}_{a}(x)$, in which every monomial appears with degree less than or equal to 9 . Note that annihilator $\tilde{N}_{a}(x)$ has a more advantageous structure than $\bar{N}_{a}(x)$ generated by Method I, since $\widetilde{N}_{a}(x)$ does not contain completely zero columns. 


\section{Examples}

360 tion method through two different discrete-time dynamic equations. The results presented in this section were computed in the Matlab environment equipped with Enhanced LFR-toolbox for Matlab [34, 27]. To model and solve semidefinite optimization (SDP) problems, YALMIP [37] with Mosek solver [38] was

$\mathrm{CPU}$ at $3.30 \mathrm{GHz}$ and $16 \mathrm{~GB}$ of RAM.

\subsection{Gradient descent}

In the literature, there exist many approaches to prove stability and convergence of the diverse alternatives of the steepest descent and other fixed-point tion mapping theorem (see e.g. [39]) and on the well-known Lyapunov theorem. In [40] a LF is considered to prove stability for a continuous-time version of the steepest descent dynamics. In [41] a multi-variable robust adaptive gradientdescent training algorithm is developed to train a recurrent neural network.

375 The convergence of the weight vector was proven using a diagonal quadratic Lyapunov function.

In this section, we consider the dynamics of the classical gradient descent algorithm in order to demonstrate the operations of the proposed method. The objective function to be minimized is chosen to be the energy (i.e. Hamiltonian) function of the Duffing oscillator. For the sake of completeness, we give the dimensionless differential equation, which describes the free motion of the undamped Duffing dynamics 42

$$
\ddot{y}-\beta y+\alpha y^{3}=0, \text { where } \beta=b^{2}, \alpha=a^{2} .
$$

If we introduce the state variables $x_{1}=y$ and $x_{2}=\dot{y}$, the Hamiltonian function of the oscillator is the following

$$
H(x)=\frac{1}{4}\left(2 x_{2}^{2}-2 b^{2} x_{1}^{2}+a^{2} x_{1}^{4}\right) .
$$


This function has two local minima in $(b / a, 0)$ and $(-b / a, 0)$ for every nonzero $a, b$ parameter values. The gradient descent dynamics for this specific objective function $H(x)$ can be given as follows:

$$
x^{+}=f(x, \varrho)=x-\varrho \cdot \nabla H(x), \varrho \in \mathcal{P}=[0.01,0.1],
$$

where $\nabla H(x)$ denotes the gradient of function $H(x)$, and $\varrho>0$ is the value of the variable step-size belonging to the given bounded interval. Furthermore, we make no restrictions on the rate of the parameter's change, the only constraint is that the value of parameter $\varrho$ in any future step should belong to the same bounded interval, namely, $\varrho^{+}=\varrho+\sigma \in \mathcal{P}$. In the computations, we let the parameter values to be $a=0.5, b=1$. Using the proposed model transformation in Section 3 the dynamics of the centered state vector $\bar{x}=\left(x_{1}+b / a, x_{2}\right)^{T}$ can be given in representation (3) required for RSD computation. The model matrices of the final simplified LFR (3) are

$$
\begin{aligned}
\left(\begin{array}{c|c}
A & B \\
\hline C & D
\end{array}\right) & =\left(\begin{array}{cc|cccc}
1 & 0 & -4 & 0 & 3 & 1 \\
0 & 1 & 0 & 1 & 0 & 0 \\
0.5 & 0 & 0 & 0 & 0 & 0 \\
0 & -1 & 0 & 0 & 0 & 0 \\
0 & 0 & 1 & 0 & 0 & 0 \\
0 & 0 & 0 & 0 & -0.5 & 0
\end{array}\right), \\
\Delta(x, \varrho) & =\left(\begin{array}{cc}
\varrho I_{2} & 0 \\
0 & x_{1} I_{2}
\end{array}\right) .
\end{aligned}
$$

From the LFR (75), we have generated vector $\pi_{b}(x, \varrho) \in \mathbb{R}^{m}$, for which a maxi- 
mal affine annihilator $N_{b}(x, \varrho) \in \mathbb{R}^{q \times m}$ is computed as proposed by [21]:

$$
\begin{aligned}
& N_{b}(x, \varrho)=\left(\begin{array}{cc|cccc}
x_{2} & -x_{1} & 0 & 0 & 0 & 0 \\
\varrho & 0 & -2 & 0 & 0 & 0 \\
0 & \varrho & 0 & 1 & 0 & 0 \\
0 & 0 & x_{1} & 0 & -1 & 0 \\
0 & 0 & x_{2} & \frac{x_{1}}{2} & 0 & 0 \\
0 & 0 & 0 & 0 & x_{1} & 2
\end{array}\right), \\
& \pi_{b}(x, \varrho)=\left(\begin{array}{ll|llll}
x_{1} & x_{2} & 0.5 \varrho x_{1} & -\varrho x_{2} & 0.5 \varrho x_{1}^{2} & -0.25 \varrho x_{1}^{3}
\end{array}\right)^{T},
\end{aligned}
$$

where the dimensions are the following: $n=2, p=4, m=n+p, q=6$. To give an LMI (9b) for the negativity of $\delta V(x, \varrho, \sigma)$, we have considered three different setups:

1. Firstly, we used the initial vector $\pi_{a}$ computed as presented in (11), which resulted in a small dimensional but conservative LMI.

2. Secondly, we generated a larger vector $\bar{\pi}_{a}$ as proposed in (58), which resulted in a less conservative LMI, but the processing time increased significantly.

3. Finally, as proposed in Method II in subsection 4.2, we used LFT and the proposed LFR simplification method to generate vector $\widetilde{\pi}_{a}$. This LMI results in the largest RSD, but the processing time increased by more than one order of magnitude compared to the case when using vector $\bar{\pi}_{a}$.

In order to compare the operations of the three models, we used three different polytopes:

$$
\begin{aligned}
& \mathcal{X}_{0}=[-3.65,-0.9] \times[-2.2,2.2], \\
& \mathcal{X}_{1}=[-4.55,-0.3] \times[-3.4,3.4], \\
& \mathcal{X}_{2}=[-5,0] \times[-4,4],
\end{aligned}
$$

for which the LMIs are solved. Table 1 summarizes the results of the optimization problems for each pair of vector $\pi_{a}$ and polytope $\mathcal{X}_{i}$. In Figure 2 and Figure 3 , the obtained RSD $\bar{\Omega}_{1, \mathcal{P}}$ with $\bar{\Omega}_{1, \mathcal{P}}^{x_{0}}$ are illustrated. Additionally, the 

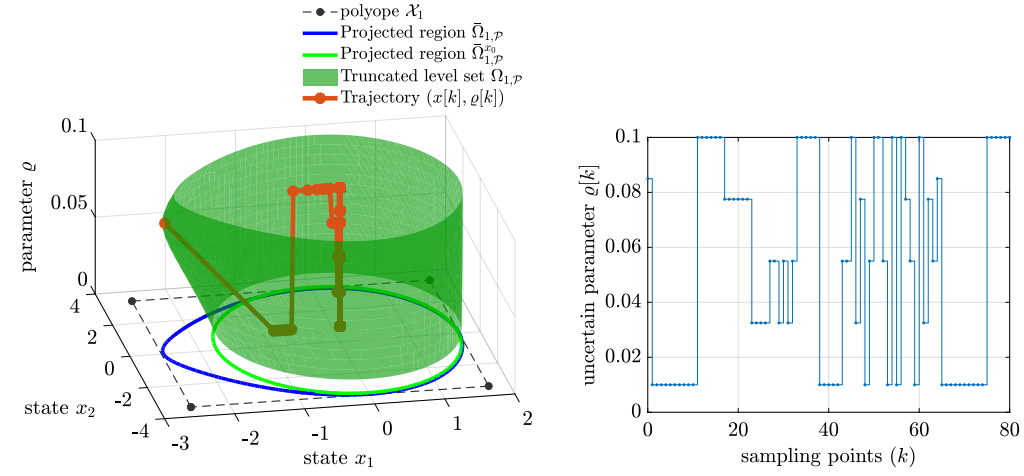

Figure 2: Trajectory $(x[k], \varrho[k])$ for the gradient-descent dynamics (left) in case of a timevarying step size $\varrho[k]$ (right). The computed truncated level set $\Omega_{1, \mathcal{P}}$ (green surface) was obtained when using vector $\bar{\pi}_{a}$ (method I) with polytope $\mathcal{X}_{1}$ (5th row of Table 1 see also Figure 3d,

truncated level set $\Omega_{1, \mathcal{P}}$ is shown in Figure 2 alongside with a possible trajectory $(x[k], \varrho[k])$.

\subsection{Uncertain Lotka-Volterra system}

We consider a 2-dimensional Lotka-Volterra (LV) model with a constant uncertain parameter $\varrho \in[1.8,2.2]$. The system equation of the $\mathrm{LV}$ model is the following:

$$
\dot{\bar{x}}_{i}=\bar{x}_{i}\left(U_{i}(\varrho) \bar{x}+b_{i}\right), \quad U(\varrho)=\left(\begin{array}{cc}
-\varrho & -3 \\
1.4 & 1
\end{array}\right), b=\left(\begin{array}{c}
5 \\
-2.4
\end{array}\right),
$$

where $U_{i}(\varrho)$ denotes the $i$ th row of the parameter dependent model matrix $U(\varrho)$, for $i=1,2$. This system has a unique nonzero equilibrium point at $x^{*}(\varrho)=-U^{-1}(\varrho) b$. In order to analyse the stability properties of $x^{*}(\varrho)$, we translate the system into this equilibrium point by introducing the centered state vector $x=\bar{x}-x^{*}(\varrho)$. One can immediately observe that the position of the equilibrium point depends on the parameter's value, therefore, this change of coordinates is also parameter dependent. Substituting the centered state vector into the system's equation, we obtain an autonomous nonlinear system:

$$
\dot{x}_{i}=\left(x_{i}+x_{i}^{*}(\varrho)\right) U_{i}(\varrho) x
$$




\begin{tabular}{|c|c|c|c|c|}
\hline set of functions, polytope & area of $\bar{\Omega}_{1, \mathcal{P}}$ & $\bar{\Omega}_{1, \mathcal{P}}^{x_{0}}$ & $\mathcal{X}_{i}$ in cubic units & average proc. time \\
\hline vector $\pi_{a}$ (initial), & 7.5909 & 8.7803 & 12.1 (in Figure $3 \mathrm{a}$ & $0.1472 \mathrm{sec}$ \\
\hline vector $\bar{\pi}_{a}(\operatorname{method} \mathrm{I}), \mathcal{X}_{0}$ & 7.7690 & 8.7122 & 12.1 (in Figure $3 \mathrm{~b}$ & $4.2773 \mathrm{sec}$ \\
\hline vector $\widetilde{\pi}_{a}(\operatorname{method} \mathrm{II}), \mathcal{X}_{0}$ & 9.2163 & 9.2168 & 12.1 (in Figure $3 c$ & $86.014 \mathrm{sec}$ \\
\hline vector $\pi_{a}$ (initial), $\quad \mathcal{X}_{1}$ & \multicolumn{3}{|c|}{ no solution found } & \\
\hline vector $\bar{\pi}_{a}(\operatorname{method} \mathrm{I}), \mathcal{X}_{1}$ & 18.2780 & 20.4107 & 28.9 (in Figure $3 \mathrm{~d}$ & $4.4903 \mathrm{sec}$ \\
\hline vector $\widetilde{\pi}_{a}($ method II $), \mathcal{X}_{1}$ & 22.1316 & 22.1516 & 28.9 (in Figure $3 \mathrm{e}$ & $112.968 \mathrm{sec}$ \\
\hline $\begin{array}{ll}\text { vector } \pi_{a} \text { (initial), } & \mathcal{X}_{2} \\
\text { vector } \bar{\pi}_{a}(\text { method I }), & \mathcal{X}_{2}\end{array}$ & \multicolumn{3}{|c|}{$\begin{array}{l}\text { no solution found } \\
\text { no solution found }\end{array}$} & \\
\hline vector $\widetilde{\pi}_{a}(\operatorname{method} \mathrm{II}), \mathcal{X}_{2}$ & 28.1455 & 28.2825 & (in Figure $3 \mathrm{f}$ & $172.517 \mathrm{sec}$ \\
\hline
\end{tabular}

Table 1: Results of the optimization problem for the three different set of rational functions $\pi_{a}, \bar{\pi}_{a}, \tilde{\pi}_{a}$, and for the three different polytopes $\mathcal{X}_{0} \subset \mathcal{X}_{1} \subset \mathcal{X}_{2}$.

\begin{tabular}{|l|c|c|c|}
\hline method & dimension of $\pi_{a}$ & dimension of its annihilator & average processing time \\
\hline initial vector $\pi_{a}$ & 10 & $8 \times 10$ & $0.1472 \mathrm{sec}$ \\
method I: $\bar{\pi}_{a}$ & 30 & $34 \times 30$ & $4.3838 \mathrm{sec}$ \\
method II: $\widetilde{\pi}_{a}$ & 52 & $102 \times 52$ & $123.833 \mathrm{sec}$ \\
\hline
\end{tabular}

Table 2: Dimension of vector $\pi_{a}$ and its corresponding annihilator with the estimated overall solver time in the three different cases. 


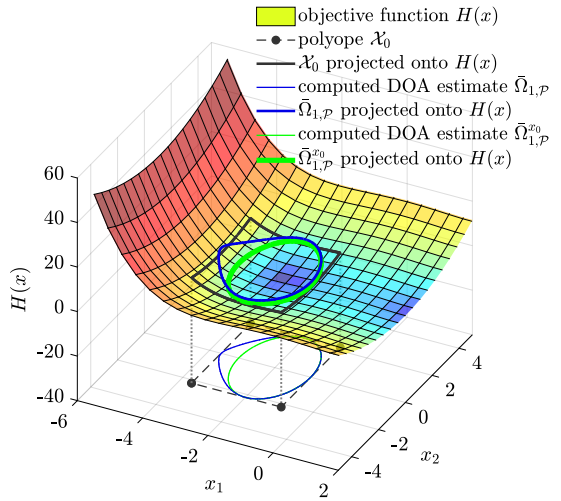

(a) Computed DOA estimate in case of $\pi_{a}$ and $\mathcal{X}_{0}$.

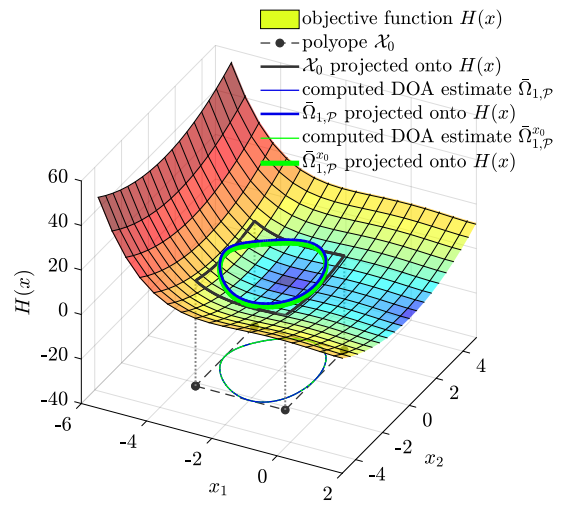

(c) Computed DOA estimate in case of $\widetilde{\pi}_{a}$ and $\mathcal{X}_{0}$.

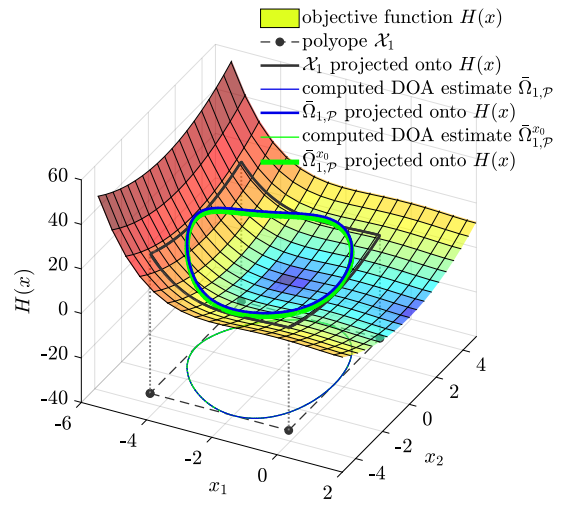

(e) Computed DOA estimate in case of $\tilde{\pi}_{a}$ and $\mathcal{X}_{1}$.

37

Figure 3: Computed DOA estimate for the classical gradient descent dynamics applied to the Hamiltonian function $H(x)$ of the Duffing oscillator (colored surface). The dashed black rectangular region illustrates polytope $\mathcal{X}$, in which the LMIs were tested. The blue and green contour lines bound the computed regions $\bar{\Omega}_{1, \mathcal{P}}$ and $\bar{\Omega}_{1, \mathcal{P}}^{x_{0}}$, respectively. In order to make the computed DOA estimate more visible, we projected $\mathcal{X}, \bar{\Omega}_{1, \mathcal{P}}$ and $\bar{\Omega}_{1, \mathcal{P}}^{x_{0}}$ onto the surface of the objective function $H(x)$. These are illustrated by the solid black, blue and green lines, 
The equation of the approximated discrete-time version for this system (using forward Euler method) is

$$
x_{i}^{+}=x_{i}+h \cdot\left(x_{i}+x_{i}^{*}(\varrho)\right) U_{i}(\varrho) x,
$$

where $h$ denotes the constant sampling period. Using the LFR-toolbox function sym2lfr, we obtain a model in the linear fractional representation $\mathcal{F}_{l}\left(A_{0}, B_{0}, C_{0}, D_{0}, \Delta_{0}\right)$. Without any further simplification procedure, the dimension of the operator $\Delta_{0}$ is $17 \times 17$.

Applying the numeric n-DOR technique [35] (implemented in the LFRtoolbox function minlfr), we obtain an LFR model $\mathcal{F}_{l}(\breve{A}, \breve{B}, \breve{C}, \breve{D}, \breve{\Delta})$ with an $8 \times 8$ dimensional operator $\breve{\Delta}$. The rounded values of the transformed model matrices are the following:

$\left(\begin{array}{ccc|ccccccccc}\breve{A} & \breve{B} \\ \hline \breve{C} & \breve{D}\end{array}\right) \simeq\left(\begin{array}{cc|cccccccc}1 & -0.0157 & 0 & 0 & 3.6021 & 0.7421 & 0 & -0.03 & 0 & 0 \\ 0.0233 & 1.0167 & 0 & 0 & -0.7294 & 3.6646 & 0 & 0 & 0 & 2.4825 \\ \hline 0.206 & 0.1472 & 0.2397 & -0.7678 & 0.5749 & 0.6412 & 0 & -0.0055 & -0.7469 & 0.3313 \\ -0.0013 & -0.0009 & -0.0015 & 0.0048 & -0.0036 & -0.004 & 0 & 0 & -0.227 & -0.0021 \\ -0.0013 & -0.0009 & 0.0002 & 0.2667 & -0.0035 & -0.0039 & 0 & 0.0019 & 0.2594 & 0.0319 \\ -0.0009 & -0.0007 & -0.0008 & 0.0531 & -0.0026 & -0.0029 & 0 & 0.0004 & 0.0516 & -0.1549 \\ -0.1416 & 0.2009 & 0.0022 & 0.5628 & -0.6181 & 0.5623 & 0.2381 & 0.004 & 0.5475 & 0.4506 \\ 0 & 1.0011 & 0.5526 & -0.0034 & -0.7395 & 3.3246 & 0.7893 & 0 & 0 & 0 \\ -0.0103 & 0 & -0.0081 & 0.0001 & -0.0341 & -0.0076 & 0.0058 & 0 & 0 & 0 \\ 0.0056 & 0.004 & 0.0067 & 0 & 0.0157 & 0.0175 & 0 & 0 & 0 & 0\end{array}\right)$,

$\breve{\Delta}=\operatorname{diag}\left(\varrho I_{5}, x_{1} I_{2}, x_{2}\right)$.

The generated set of rational functions can be computed as follows: $\breve{\pi}=\left(I_{8}-\right.$ $\breve{\Delta} \breve{D})^{-1} \breve{\Delta} \breve{C} x$. Due to the numerical floating point operations of the n-DOR, the symbolic expression of vector $\breve{\pi}$ is exceedingly lengthy and complicated.

On the other hand, using the proposed symbolic LFR simplification proce- 
dure proposed in Section 3 , we can generate the following LFR model:

\begin{tabular}{|c|c|c|c|c|c|c|c|c|c|c|c|c|}
\hline & 1 & -0.0157 & $\mid 1$ & 0 & -5 & 0.15 & 0.0786 & 0 & 0 & 0.03 & 0 \\
\hline & & 0.0233 & 1.0167 & 0 & 0 & 0.0513 & -0.07 & -5.0833 & 1.0167 & 0 & -0.014 & -4.27 \\
\hline & & -0.2433 & 0 & 0 & 1 & 1.2167 & 0 & 0 & 0 & 1 & 0 & 0 \\
\hline & & 0 & 0 & 0 & 0 & 0 & 0 & 0 & 0 & -0.2381 & 0 & 0 \\
\hline \multirow{7}{*}{$\left(\begin{array}{l}A \\
C\end{array}\right.$} & \multirow{7}{*}{$\simeq$} & -0.0476 & 0 & 0 & 0 & 0.2381 & 0 & 0 & 0 & 0 & 0 & 0 \\
\hline & & 0 & 0 & 0 & 0 & 0 & 0 & 0 & 0 & 0 & -0.0476 & 0 \\
\hline & & 0 & -0.0476 & 0 & 0 & 0 & 0 & 0.2381 & 0 & 0 & 0 & 0 \\
\hline & & 0 & -0.2398 & 0 & 0 & 0 & 0 & 1.1991 & 0 & 0 & 0 & 1 \\
\hline & & -0.01 & 0 & 0 & 0 & 0.05 & 0 & 0 & 0 & 0 & 0 & 0 \\
\hline & & 0 & -1 & 0 & 0 & 0 & 0 & 5 & 0 & 0 & 0 & 0 \\
\hline & & 0 & -0.0023 & 0 & 0 & 0 & 0 & 0.0117 & 0 & 0 & 0 & 0 \\
\hline$=$ & $\operatorname{diag}(\varrho$ & $2, x$ & & & & & & & & & $(82)$ & \\
\hline
\end{tabular}

The sparse structure of matrices $A, B, C$, and $D$ makes possible to obtain a symbolically well-tractable explicit expression for the rational vector $\pi$ :

$$
\pi=\frac{1}{5 \varrho-21} \cdot\left(\begin{array}{c}
0.01 \varrho x_{1}\left(21 x_{1}-5 \varrho x_{1}+511\right) \\
-0.05 \varrho x_{1}^{2} \\
\varrho x_{1} \\
-\varrho x_{1} x_{2} \\
\varrho x_{2} \\
0.009836 \varrho x_{2}\left(5 x_{2}+512\right) \\
0.21 x_{1}^{2} \\
21 x_{1} x_{2} \\
0.04918 x_{2}^{2}
\end{array}\right)
$$

In Table 3, one can see the runtime results of the optimization procedure obtained for both models $\mathcal{F}_{l}(\breve{A}, \breve{B}, \breve{C}, \breve{D}, \breve{\Delta})$ and $\mathcal{F}_{l}(A, B, C, D, \Delta)$, the first obtained by the numeric n-DOR technique and the second by our LFR simplification technique. In order to reduce the conservatism of the second LMI 9b), in both cases we used the augmented vector $\bar{\pi}_{a}$ proposed in Method I. In Figure 4 and 5 , we illustrate the obtained RSD of the locally stable fixed-point. 

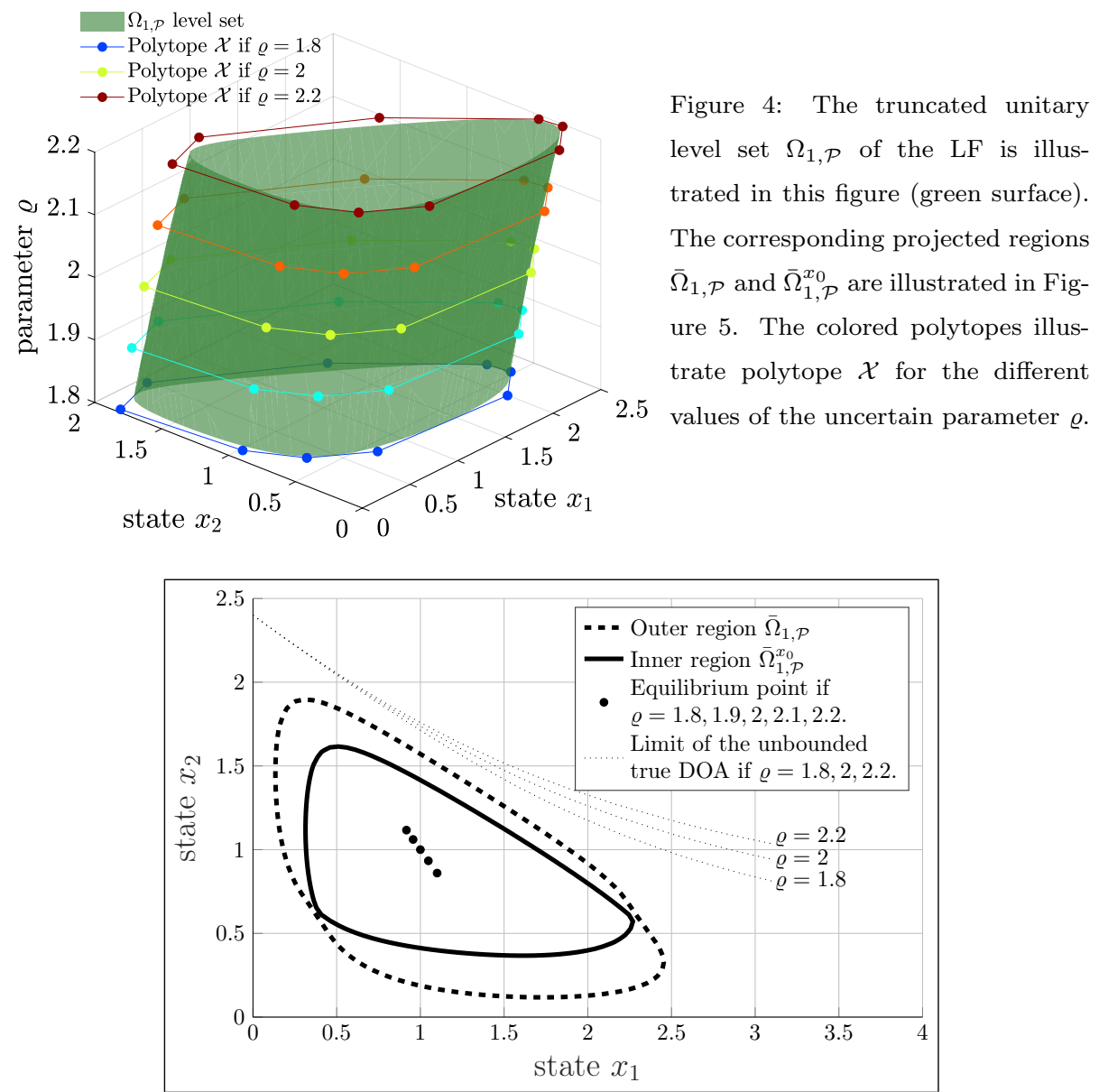

Figure 5: The robust stability domain $\bar{\Omega}_{1, \mathcal{P}}^{x_{0}}$ of the unique non-trivial parameter dependent fixed-point $x^{*}(\varrho)$ of the discretized Lotka-Volterra model is illustrated in this figure (solid line) alongside with $\bar{\Omega}_{1, \mathcal{P}}$ (dashed line). The position of the fixed-point $x^{*}(\varrho)$ is illustrated for a few values of the uncertain parameter $\varrho$. Furthermore, the approximated boundary of the (unbounded) true DOA is illustrated by the thin dotted lines corresponding to three different values of the uncertain parameter $\varrho$. The approximate is computed by simulating the time inverted continuous-time model. 


\begin{tabular}{|l|cc|c|}
\hline used model & area of $\bar{\Omega}_{1, \mathcal{P}}$ and & $\bar{\Omega}_{1, \mathcal{P}}^{x_{0}}$ in cubic units & processing time \\
\hline initial: $\mathcal{F}_{l}\left(A_{0}, B_{0}, C_{0}, D_{0}, \Delta_{0}\right)$ & solver did not terminate successfully & \\
n-DOR: $\mathcal{F}_{l}(\breve{A}, \breve{B}, \breve{C}, \breve{D}, \breve{\Delta})$ & 1.3037 & 2.1651 & $\sim 1800 \mathrm{sec}$ \\
simplified LFR: $\mathcal{F}_{l}(A, B, C, D, \Delta)$ & 1.5019 & 2.4747 & $\sim 450 \mathrm{sec}$ \\
\hline
\end{tabular}

Table 3: Results of the optimization problem (areas of the obtained regions and processing time), when using n-DOR and our proposed LFR simplification method to generate a smaller LFR.

\subsection{Three dimensional rational model}

In the following third order rational system taken from [18, 21, we can compare the discrete-time DOA estimate with the continuous-time case. The continuous-time model of the system is:

$$
\dot{x}=f(x), \text { where } f(x)=\left(\begin{array}{c}
x_{2}+e x_{3}+\frac{e x_{1}}{x_{2}^{2}+1} \\
-x_{1}-x_{2}+e x_{1}^{2} \\
e\left(-2 x_{1}-2 x_{3}-x_{1}^{2}\right)
\end{array}\right) \text {, and } e=\frac{1}{2} .
$$

Using forward Euler method, we can give a discrete-time model for this system:

$$
x^{+}=x+h f(x)
$$

where $h$ denotes the constant sampling period. In the computations, we used $h=0.1$. After the symbolic model simplifications, the nonlinear terms in vector $\pi(x, \varrho)$ are the following (for both $\mathrm{CT}$ and DT models):

$$
\pi(x, \varrho)=\left(\begin{array}{lll}
x_{1}^{2} & \frac{x_{1} x_{2}^{2}}{x_{2}^{2}+1} & \frac{x_{1} x_{2}}{x_{2}^{2}+1}
\end{array}\right)^{T} .
$$

Vector $\pi_{a}(x, \varrho, \sigma)$ for the LMI $9 \mathrm{~b}$ are given differently for the CT and DT cases: $\pi_{a}^{(\mathrm{DT})}(x, \varrho, \sigma)$ computed as presented in Eq. (11) in the DT case, and

$$
\pi_{a}^{(\mathrm{CT})}(x, \varrho, \sigma)=\left(\begin{array}{c}
\pi_{b}(x, \varrho) \\
\frac{\partial \pi}{\partial x} f(x, \varrho)+\frac{\partial \pi}{\partial \varrho} \sigma
\end{array}\right)
$$

in the $\mathrm{CT}$ case, where $\sigma$ denotes the time derivative of $\delta$ in this case. In order to obtain a larger estimate, the obtained rational terms in both $\pi_{a}^{(\mathrm{CT})}(x, \varrho, \sigma)$ 
and $\pi_{a}^{(\mathrm{DT})}(x, \varrho, \sigma)$ were supplemented with additional new rational terms as proposed in Section 4.1 (Method I) and Section 4.1 (Method II). In order to be able to evaluate the results of the operations in the four different cases, we used a common polytope for all cases:

$$
\mathcal{X}=[-3.771,3.5195] \times[-4.6077,5.1943] \times[-8.4274,6.7204] .
$$

The results of the RSD computation (including the volume of the computed

required for computational DOA estimation and to generate uncertain rational terms, which will give the structure of the parameterized Lyapunov function. 


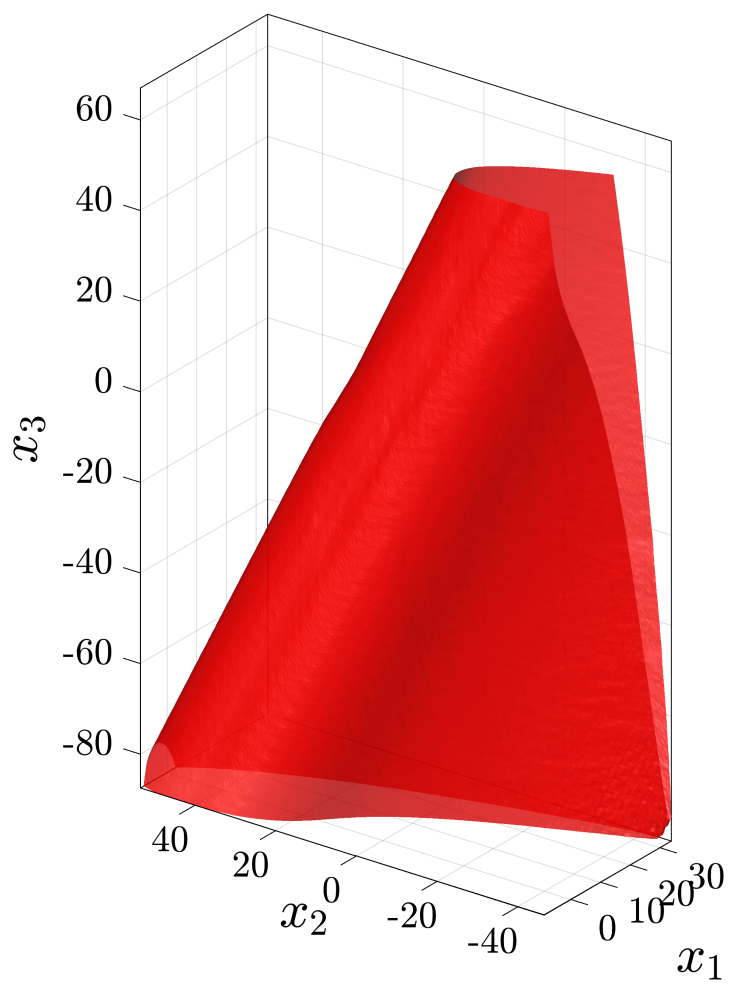

Figure 6: Numerically computed true DOA of the 3rd order rational system.

\begin{tabular}{|l|l|l|l|c|c|c|r|}
\hline 3D rational model & $m$ & $m_{a}$ & \#new terms & \#vars & volume & solver time \\
\hline CT + Method I. (Fig. & 7 & 6 & 27 & 18 & 1926 & 314.479 & $4.758 \mathrm{sec}$ \\
CT + Method II. (Fig. & 9 & 6 & 21 & 12 & 1575 & 304.804 & $3.349 \mathrm{sec}$ \\
\hline DT + Method I. (Fig. & 8 & 6 & 36 & 27 & 2223 & 279.382 & $8.401 \mathrm{sec}$ \\
DT + Method II. (Fig. 10 & 6 & 54 & 45 & 6705 & 318.521 & $247.143 \mathrm{sec}$ \\
\hline
\end{tabular}

Table 4: Results of the RSD computation for the continuous-time and discrete-time models using both tuning-knobs described in Section 4.1 and Section 4.2 Column $m_{a}$ contains the number of the overall rational terms in the second LMI conditions $9 \mathrm{~b}$, and it also gives the size of the second LMI. The 4th column gives the number of the new rational terms, which were introduced by Method I or II. In the 5th column we present the number of free decision variables of the optimization problem. The last column presents the processing time of the semidefinite optimization solver. 


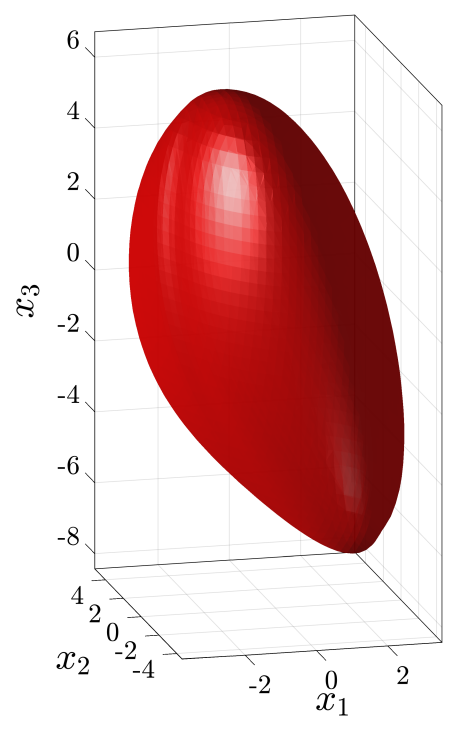

Figure 7: Computed RSD estimate for the CT model using the CT version of Method I of Section 4.1 proposed in [18 Theorem 4.1]. Volume: 314.479 cubic units.

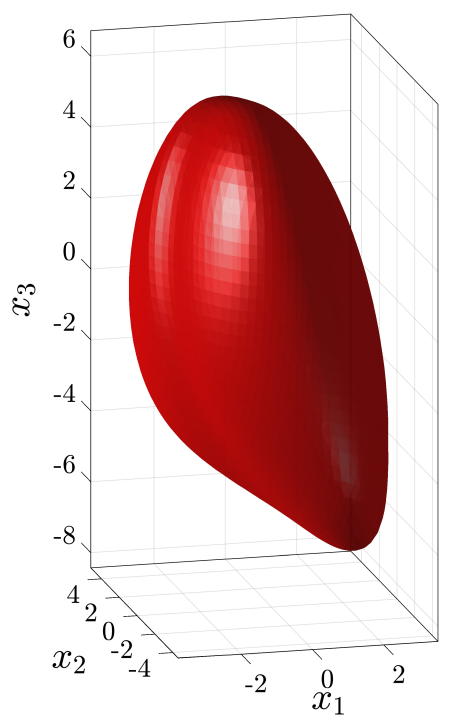

Figure 9: Computed RSD estimate for the CT model using Method II of Section 4.2 Volume: 304.804 cubic units.

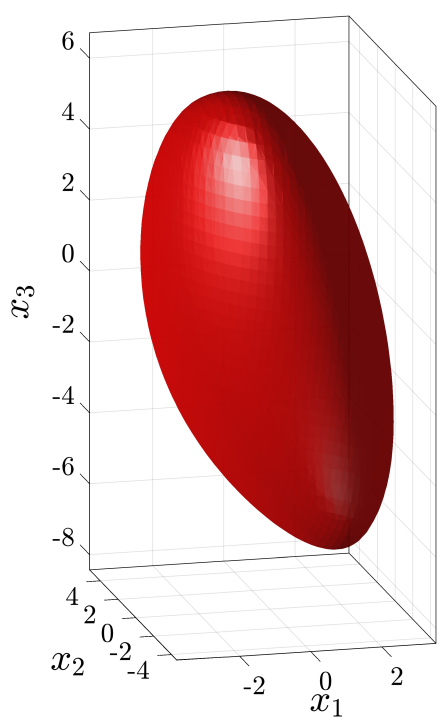

Figure 8: Computed RSD estimate for the DT model using Method I of Section 4.1 Volume: 279.382 cubic units.

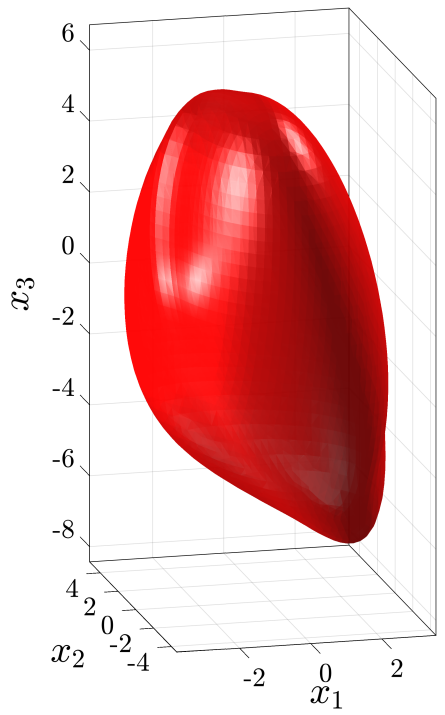

Figure 10: Computed RSD estimate for the DT model using Method II of Section 4.2 Volume: 318.521 cubic units. 
The proposed linear fractional representation (LFR) simplification method is based on our earlier results introduced in [22, Compared to the numeric ndimensional order reduction (n-DOR) technique, the proposed LFR simplification method may result in a higher dimensional LFR (with a larger block $\Delta$ ), but it keeps the transformed model matrices in a symbolically more tractable form. Furthermore, we showed that the simplified smaller model gives the same LF with the same computed DOA, but obtained by solving a smaller LMI problem with less number of decision variables. Differently from n-DOR, our symbolic model simplification procedure was shown to keep the sparse matrix structure of the model matrices $(A, B, C, D)$ of the initial LFR model, therefore, the obtained smaller dimensional model matrices will generate a reduced number of symbolically more tractable rational functions. The simplified expressions of the rational functions in $\pi_{b}$ are also advantageous when a maximal annihilator is generated for vector $\pi_{b}$. Additionally, we have proposed two tuning heuristics to obtain less conservative LMI conditions for the negativity of difference of the Lyapunov function.

\section{Acknowledgements}

We gratefully acknowledge the support of the National Research, Development and Innovation Office - NKFIH through grant OTKA 125739. The research was also supported by Pázmány Péter Catholic University and by the Ministry of National Economy of Hungary through the projects KAP-1.1-17 and GINOP-2.3.2-15-2016-00002, respectively. The second author gratefully acknowledges the research grants supported by the János Bolyai Research Scholarship of the Hungarian Academy of Sciences.

\section{References}

[1] F. Camilli, L. Grüne, F. Wirth, Control Lyapunov functions and Zubov's method, SIAM Journal on Control and Optimization 47 (1) (2008) 301-326. 
[2] A. Vannelli, M. Vidyasagar, Maximal Lyapunov functions and domains of attraction for autonomous nonlinear systems, Automatica 21 (1) (1985) 69-80. doi:10.1016/0005-1098(85)90099-8.

[3] S. Rozgonyi, K. M. Hangos, G. Szederkényi, Determining the domain of attraction of hybrid non-linear systems using maximal Lyapunov functions, Kybernetika 46 (2010) 19-37. doi:10338.dmlcz/140048.

[4] Y. Ohta, H. Imanishi, L. Gong, H. Haneda, Computer generated Lyapunov functions for a class of nonlinear systems, IEEE Transactions on Circuits and Systems 40 (1993) 343-354. doi:10.1109/SSST.1989.72503.

[5] P. Giesl, S. Hafstein, Construction of Lyapunov functions for nonlinear planar systems by linear programming, Journal of Mathematical Analysis and Applications 388 (2012) 463-479. doi:10.1016/j · jmaa.2011.10.047.

[6] R. Bouyekhf, L. T. Gruyitch, An alternative approach for stability analysis of discrete time nonlinear dynamical systems, Journal of Difference Equa1 tions and Applications 24 (1) (2018) 68-81. arXiv:https://doi.org/10. 1080/10236198.2017.1391239, doi:10.1080/10236198.2017.1391239.

[7] D. P. Mandic, J. Chambers, Recurrent Neural Networks for Prediction: Learning Algorithms, Architectures and Stability, John Wiley \& Sons, Inc., New York, NY, USA, 2001.

[8] H. L. Smith, H. R. Thieme, Persistence and global stability for a class of discrete time structured population models, Discrete and Continous Dynamtical Systems Series A (DCDS-A)doi:10.3934/dcds.2013.33.xx.

[9] J. Kapinski, J. V. Deshmukh, S. Sankaranarayanan, N. Arechiga, Simulation-guided Lyapunov analysis for hybrid dynamical systems, in: Proceedings of the $17^{\text {th }}$ international conference on Hybrid systems: computation and control, ACM, 2014, pp. 133-142. 
[10] J. Kapinski, J. Deshmukh, Discovering forward invariant sets for nonlinear dynamical systems, Vol. 117, 2015, pp. 259-264. doi:10.1007/ 978-3-319-12307-3_37.

[11] R. Bobiti, M. Lazar, A sampling approach to finding Lyapunov functions for nonlinear discrete-time systems, in: 2016 European Control Conference (ECC), 2016, pp. 561-566. doi:10.1109/ECC. 2016.7810344.

[12] G. Chesi, Rational lyapunov functions for estimating and controlling the robust domain of attraction, Automatica 49 (4) (2013) 1051-1057.

[13] C. W. Scherer, S. Weiland, Linear Matrix Inequalities In Control, Technical University of Delft, The Netherlands, Delft, 2000.

[14] S. Boyd, L. Vandenberghe, Convex Optimization, Cambridge University Press, New York, NY, USA, 2004.

[15] K. Zhou, J. C. Doyle, K. Glover, et al., Robust and optimal control, Vol. 40, Prentice hall New Jersey, 1996.

[16] L. E. Ghaoui, G. Scorletti, Control of rational systems using linearfractional representations and linear matrix inequalities, Automatica 32 (9) (1996) 1273-1284. doi:10.1016/0005-1098(96)00071-4

[17] D. Coutinho, M. Fu, A. Trofino, Regional stability and performance analysis for a class of nonlinear discrete-time systems, Vol. 3, Las Vegas, NV, 2002, pp. 2675-2680.

[18] A. Trofino, T. J. M. Dezuo, LMI stability conditions for uncertain rational nonlinear systems, International Journal of Robust and Nonlinear Control 24 (18) (2013) 3124-3169. doi:10.1002/rnc.3047.

[19] B. Bhiri, C. Delatre, M. Zasadzinski, K. Abderrahim, Finite-time stability via polynomial Lyapunov function of nonlinear quadratic systems, Vol. 2016-July, 2016, pp. 1142-1147. doi:10.1109/ACC.2016.7525069 
[20] D. Han, M. Althoff, On estimating the robust domain of attraction for uncertain non-polynomial systems: An LMI approach, 2016, pp. 21762183. doi:10.1109/CDC.2016.7798586.

[26] G. Chesi, Domain of attraction: analysis and control via SOS program-

[27] J.-F. Magni, User Manual of the Linear Fractional Representation Toolbox:

[28] S. Moheimani (Ed.), Perspectives in Robust Control, Vol. 268, SpringerVerlag New York, 2001. doi:10.1007/BFb0110609. 
[29] L. F. Buzachero, E. Assuncao, E. R. Da Silva, M. C. Teixeira, New techniques for optimizing the norm of robust controllers of polytopic uncertain linear systems, INTECH Open Access Publisher, 2012. doi: $10.5772 / 38920$

[30] A. Marcos, D. G. Bates, I. Postlethwaite, A multivariate polynomial matrix order-reduction algorithm for linear fractional transformation modelling, IFAC Proceedings Volumes 38 (1) (2005) 327-332, $16^{\text {th }}$ IFAC World Congress. doi:10.3182/20050703-6-CZ-1902.00999.

[31] S. Hecker, A. Varga, Symbolic techniques for low order LFT-modelling (Mar. 2005).

[32] S. Hecker, A. Varga, Symbolic manipulation techniques for low order LFTbased parametric uncertainty modelling, International Journal of Control 79 (11) (2006) 1485-1494. doi:10.1080/00207170600725644.

[33] S. Hecker, Improved mu-analysis results by using low-order uncertainty modeling techniques, Journal of guidance, control, and dynamics 31 (4) (2008) 962-969.

[34] S. Hecker, A. Varga, J.-F. Magni, Enhanced LFR-toolbox for MATLAB, 2004, pp. 25-29. doi:10.1109/CACSD.2004.1393845

[35] R. D'Andrea, S. Khatri, Kalman decomposition of linear fractional transformation representations and minimality, in: Proceedings of the 1997 American Control Conference (Cat. No.97CH36041), Vol. 6, 1997, pp. 3557-3561 vol.6. doi:10.1109/ACC.1997.609484.

[36] J. C. Doyle, F. Paganini, R. D'Andrea, S. Khatri, Approximate behaviors, in: Decision and Control, 1996., Proceedings of the $35^{\text {th }}$ IEEE Conference on, Vol. 1, 1996, pp. 688-693 vol.1. doi:10.1109/CDC.1996.574430.

[37] J. Löfberg, Yalmip : A toolbox for modeling and optimization in MATLAB, 4 in: Proceedings of the CACSD Conference, Taipei, Taiwan, 2004. doi: 565 10.1109/CACSD. 2004.1393890. 
[38] MOSEK ApS, The MOSEK optimization toolbox for MATLAB manual. Version 7.1 (Revision 28). (2015).

URL http://docs.mosek.com

[39] A. R. Heravi, G. A. Hodtani, A new robust fixed-point algorithm and its convergence analysis, Journal of Fixed Point Theory and Applications 19 (4) (2017) 3191-3215. doi:10.1007/s11784-017-0474-5.

[40] N. Qian, On the momentum term in gradient descent learning algorithms, Neural networks 12 (1) (1998) 145-151.

[41] Z. Xu, Q. Song, D. Wang, Recurrent neural tracking control based on multivariable robust adaptive gradient-descent training algorithm, Neural Computing and Applications 21 (7) (2012) 1745-1755. doi:10.1007/ s00521-011-0618-2.

[42] S. Lenci, G. Rega, Forced harmonic vibration in a duffing oscillator with negative linear stiffness and linear viscous damping, The Duffing Equation: Nonlinear Oscillators and their Behaviour (eds. I. Kovacic and MJ Brennan), Wiley, ISBN 830182507 (2011) 219-276. 